\title{
Using biogeochemical data assimilation to assess the relative skill of multiple ecosystem models in the Mid-Atlantic Bight: effects of increasing the complexity of the planktonic food web
}

\author{
Y. Xiao and M. A. M. Friedrichs \\ Virginia Institute of Marine Science, College of William \& Mary, P.O. Box 1346, Gloucester Point, VA 23062, USA \\ Correspondence to: M. A. M. Friedrichs (marjy@vims.edu)
}

Received: 8 December 2013 - Published in Biogeosciences Discuss.: 8 January 2014

Revised: 4 April 2014 - Accepted: 20 April 2014 - Published: 6 June 2014

Abstract. Now that regional circulation patterns can be reasonably well reproduced by ocean circulation models, significant effort is being directed toward incorporating complex food webs into these models, many of which now routinely include multiple phytoplankton $(\mathrm{P})$ and zooplankton (Z) compartments. This study quantitatively assesses how the number of phytoplankton and zooplankton compartments affects the ability of a lower-trophic-level ecosystem model to reproduce and predict observed patterns in surface chlorophyll and particulate organic carbon. Five ecosystem model variants are implemented in a one-dimensional assimilative (variational adjoint) model testbed in the Mid-Atlantic Bight. The five models are identical except for variations in the level of complexity included in the lower trophic levels, which range from a simple $1 \mathrm{P} 1 \mathrm{Z}$ food web to a considerably more complex 3P2Z food web. The five models assimilated satellite-derived chlorophyll and particulate organic carbon concentrations at four continental shelf sites, and the resulting optimal parameters were tested at five independent sites in a cross-validation experiment. Although all five models showed improvements in model-data misfits after assimilation, overall the moderately complex $2 \mathrm{P} 2 \mathrm{Z}$ model was associated with the highest model skill. Additional experiments were conducted in which $20 \%$ random noise was added to the satellite data prior to assimilation. The $1 \mathrm{P}$ and $2 \mathrm{P}$ models successfully reproduced nearly identical optimal parameters regardless of whether or not noise was added to the assimilated data, suggesting that random noise inherent in satellitederived data does not pose a significant problem to the assimilation of satellite data into these models. However, the most complex model tested (3P2Z) was sensitive to the level of random noise added to the data prior to assimilation, high- lighting the potential danger of over-tuning inherent in such complex models.

\section{Introduction}

In spite of recent advances in marine ecosystem modeling that now allow for the incorporation of multiple plankton functional types and/or size classes (e.g., Follows et al., 2007; Kishi et al., 2007; Salihoglu and Hofmann, 2007), it remains ambiguous as to whether models with additional plankton compartments necessarily perform better than models characterized by relatively simple structures. Although the use of a single plankton compartment may fail to resolve key processes in a given ecosystem (e.g., Ward et al., 2013), the inclusion of additional complexity in plankton structure comes with a substantial cost: significant uncertainties will inevitably be associated with the additional state variables and required parameters (Anderson, 2005; Flynn, 2005). Hence these trade-offs in model structure selection pose a challenging question: how does one determine how many phytoplankton and zooplankton compartments need to be included in a given application of a lower trophic model?

Multiple model comparison studies have helped improve our understanding of the trade-offs of increasing ecosystem model complexity, yet many of these studies have not directly isolated the effects of increasing plankton complexity (e.g., Baird and Suthers, 2010; Costanza and Sklar, 1985; Fulton et al., 2003; Hannah et al., 2010; Paudel and Jawitz, 2012; Raick et al., 2006). For example, a recent community data assimilative modeling comparison exercise (Friedrichs et al., 
2007) revealed that ecosystem models with multiple phytoplankton $(\mathrm{P})$ state variables were quantitatively more skillful (in terms of reproducing observations of chlorophyll, primary production, export and nitrate at multiple sites) than models with single P compartments. However, the 12 models participating in the Friedrichs et al. (2007) comparison study varied in many different ways, including nutrient limitations, variable elemental compositions and zooplankton $(\mathrm{Z})$ state variables, making it difficult to determine why certain models performed better than others. Lehmann et al. (2009) compared two models with different numbers of plankton compartments (1P1Z with $2 \mathrm{P} 1 \mathrm{Z}$ ) and concluded that the additional phytoplankton state variable improved model skill. However, in this case it was not completely clear whether the improvement was due to the additional phytoplankton compartment or was caused by other differences in the structures of the two models such as the variable carbon: nitrogen ratio included in the more complex model. Likewise, Hashioka et al. (2012) evaluated the role of functional groups in four global ecosystem models. Although differences in model performance were found, these were largely attributed to variations in underlying governing mechanisms, and not necessarily to differences in the numbers and specific characteristics of each model's phytoplankton functional types.

In contrast to these previous efforts that compared models that varied in many ways based on different assumptions made by different investigators, Bagniewski et al. (2011) compared the relative skill of three models that differed only in their formulations for fast-sinking diatom aggregates and cysts. Although none of their models could be rejected based on misfit with available observations, the inclusion of export by diatom aggregation was found to be a process that significantly improved model-data fit. In the study presented here, the focus is on the inter-model differences induced solely by variations in the assumed phytoplankton and zooplankton structures. In other words, the five ecosystem models tested in this study are identical except for variations in the level of complexity included in the $\mathrm{P}$ and $\mathrm{Z}$ compartments, and range from a simple $1 \mathrm{P} 1 \mathrm{Z}$ to a considerably more complex 3P2Z food web. To further simplify the comparison, functional types were not considered, but instead, the multiple $\mathrm{P}$ and $\mathrm{Z}$ only account for size class differences.

Here relative model skill is defined as how well the models represent observations over a specified range of conditions, or, more practically, how well the models fit the data (Jolliff et al., 2009; Stow et al., 2009, Friedrichs et al., 2009). Since ecosystem model performance is very sensitive to the arbitrary choice of ecological parameter values (Rykiel, 1996), it is critical to rigorously optimize the parameter values of individual models prior to comparing their relative skill in order to insure that innate differences in model structures are being compared, rather than the degree of subjective tuning (Friedrichs et al., 2006). Thus in this analysis each of the five models was parameterized in a 1-D assimilative framework, and parameters were optimized through the assimilation of satellite-derived data. In this way, all five models were compared at their optimum skill. In addition, because all models were forced with identical physics, the difference in model performance was only a function of the varying $\mathrm{P}$ and $\mathrm{Z}$ food web structures.

The objective of this study is not to identify a model with the highest possible skill in this particular region of the ocean, but rather the goal is to determine how varying the number of plankton variables within a given model affects model performance. In other words, this study examines how model skill, specifically skill in reproducing surface chlorophyll and particulate organic carbon concentrations, is affected by manipulating the complexity of the planktonic food web without altering other underlying formulations and assumptions in the model.

\section{Methods}

\subsection{Ecosystem models}

In this study five nitrogen-based marine ecosystem models were compared. All are nitrogen-phytoplanktonzooplankton-detritus (NPZD)-type models incorporating identical biogeochemical processes (as described in Fennel et al. 2006), with the only difference between models being the number of phytoplankton and zooplankton groups: $1 \mathrm{P} 1 \mathrm{Z}, 2 \mathrm{P} 1 \mathrm{Z}, 2 \mathrm{P} 2 \mathrm{Z}$ and $3 \mathrm{P} 1 \mathrm{Z}$ and $3 \mathrm{P} 2 \mathrm{Z}$ food webs. The most complex 3P2Z model includes three P compartments (pico-, nano- and micro-phytoplankton) with three corresponding chlorophyll state variables and two $\mathrm{Z}$ compartments (microand meso-zooplankton). In the simplest $1 \mathrm{P} 1 \mathrm{Z}$ model, the single $\mathrm{P}$ and $\mathrm{Z}$ compartments represent an average of three phytoplankton size classes and micro-zooplankton, respectively. In the $2 \mathrm{P}$ models, one phytoplankton compartment represents the micro-phytoplankton and one represents an average of pico- plus nano-phytoplankton. The key parameters that differentiate $\mathrm{P}$ size classes include maximum chlorophyll-tocarbon ratios, nutrient half-saturation constants, maximum growth rates and sinking rates, whereas $\mathrm{Z}$ compartments vary in grazing rates and food preference. Both micro- and meso-zooplankton were assumed to graze on all phytoplankton size classes but with varying grazing rates. This allowed micro-zooplankton to prefer pico- and nano-phytoplankton, whereas meso-zooplankton preferred micro-phytoplankton. A summarized list of critical parameters for the various plankton state variables is provided in Table 1 and the biological equations are provided in the Appendix.

Each of the five marine ecosystem models were embedded in a 1-D (vertical) physical model that contains standard parameterizations for vertical advection, diffusion and sinking particles that have been thoroughly described in a number of other 1-D modeling studies (Friedrichs et al., 2007; Ward et al., 2010; Xiao and Friedrichs, 2014). Initial and bottom boundary conditions for the model state variables were set 
Table 1. Key parameters that differentiate the phytoplankton $(\mathrm{P})$ and zooplankton $(\mathrm{Z})$ size classes.

\begin{tabular}{|c|c|c|c|c|c|c|c|}
\hline Parameter & Symbol & $\mathrm{P}$ size class* & $1 \mathrm{P} 1 \mathrm{Z}$ & $2 \mathrm{P} 1 \mathrm{Z}$ & $2 \mathrm{P} 2 \mathrm{Z}$ & 3P1Z & $3 \mathrm{P} 2 \mathrm{Z}$ \\
\hline \multirow{3}{*}{$\begin{array}{l}\text { Maximum Chl : C ratio } \\
\left((\mathrm{mg} \mathrm{Chl})\left(\mathrm{mg} \mathrm{C}^{-1}\right)\right.\end{array}$} & $\theta_{\text {max_SP }}$ & Small & 0.05 & 0.03 & 0.03 & 0.03 & 0.03 \\
\hline & $\theta_{\mathrm{max} \_\mathrm{MP}}$ & Medium & & & & 0.05 & 0.05 \\
\hline & $\theta_{\mathrm{max} \_L P}$ & Large & & 0.06 & 0.06 & 0.06 & 0.06 \\
\hline \multirow{3}{*}{$\begin{array}{l}\text { Half saturation for } \mathrm{NO}_{3} \text { uptake } \\
(\mathrm{mmol} \mathrm{N} \mathrm{m}-3)\end{array}$} & $k_{\mathrm{NO} 3 \_\mathrm{SP}}$ & Small & 0.5 & 1.0 & 1.0 & 1.0 & 1.0 \\
\hline & $k_{\mathrm{NO} 3 \_\mathrm{MP}}$ & Medium & & & & 1.5 & 1.5 \\
\hline & $k_{\mathrm{NO} 3 \_\mathrm{LP}}$ & Large & & 1.5 & 1.5 & 2.0 & 2.0 \\
\hline \multirow{3}{*}{$\begin{array}{l}\text { P maximum growth rate } \\
\left(d^{-1}\right)\end{array}$} & $\mu_{\mathrm{max} \_\mathrm{SP}}$ & Small & 1.0 & 1.8 & 1.8 & 1.5 & 1.5 \\
\hline & $\mu_{\mathrm{max} \_\mathrm{MP}}$ & Medium & & & & 1.2 & 1.2 \\
\hline & $\mu_{\mathrm{max} \_\mathrm{LP}}$ & Large & & 1.0 & 1.0 & 1.0 & 1.0 \\
\hline \multirow[t]{3}{*}{$P$ sinking rate $\left[d^{-1}\right]$} & $w_{\mathrm{SP}}$ & Small & 0.1 & 0.1 & 0.1 & 0.1 & 0.1 \\
\hline & $w_{\mathrm{MP}}$ & Medium & & & & 0.2 & 0.2 \\
\hline & $w_{\mathrm{LP}}$ & Large & & 0.4 & 0.4 & 0.4 & 0.4 \\
\hline \multirow{3}{*}{$\begin{array}{l}\text { Micro-Z grazing rate } \\
\left(d^{-1}\right)\end{array}$} & $g_{\mathrm{SP} \_S Z}$ & Small & 0.6 & 0.8 & 1.2 & 0.4 & 1.0 \\
\hline & $g_{\mathrm{MP} \_S Z}$ & Medium & & & & 0.8 & 0.6 \\
\hline & $g_{\mathrm{LP}}{ }_{\mathrm{SZ}}$ & Large & & 1.2 & 0.4 & 1.0 & 0.2 \\
\hline \multirow{3}{*}{$\begin{array}{l}\text { Meso- } Z \text { grazing rate } \\
\left(d^{-1}\right)\end{array}$} & $g_{\mathrm{SP} \_L Z}$ & Small & & & 0.4 & & 0.2 \\
\hline & $g_{\mathrm{MP} \_L Z}$ & Medium & & & & & 0.6 \\
\hline & $g_{\mathrm{LP} \_L Z}$ & Large & & & 1.2 & & 1.0 \\
\hline Micro-Z basal metabolism rate $\left(\mathrm{d}^{-1}\right)$ & $l_{\mathrm{BM} \_\mathrm{SZ}}$ & & 0.1 & 0.1 & 0.1 & 0.1 & 0.1 \\
\hline Meso- $Z$ basal metabolism rate $\left(\mathrm{d}^{-1}\right)$ & $l_{\mathrm{BM} \_\mathrm{LZ}}$ & & & & 0.1 & & 0.1 \\
\hline
\end{tabular}

* The three size classes represent pico-, nano- and micro-phytoplankton (small, medium and large).

the same as in Xiao and Friedrichs (2014), i.e., provided by a corresponding three-dimensional (3-D) 1P1Z model implementation (Hofmann et al., 2008; 2011). Models with two size classes were initialized as one-half of the 3-D 1P1Z concentrations, and models with three size classes were initialized as one-third of these concentrations. Sensitivity experiments demonstrated that the 1-D models were not sensitive to these initial size fractionation ratios. In all experiments, carbon was derived by converting nitrogen $(\mathrm{N})$ to carbon $(\mathrm{C})$ via a constant Redfield $\mathrm{C}: \mathrm{N}$ ratio and model estimates of particulate organic carbon (POC) were computed as the sum of all phytoplankton, zooplankton and detritus. All five models were run from 1 January 2004 through 31 December 2004 with a time step of $1 \mathrm{~h}$.

\subsection{Satellite-derived data}

Based on the results of Xiao and Friedrichs (2014), three types of daily $9 \mathrm{~km}$ data were derived from the Sea-viewing Wide Field-of-view Sensor (SeaWiFS) and assimilated into the five models described above (Table 2): size-fractionated chlorophyll $a$ (Pan et al., 2010), total chlorophyll $a$ (computed as the sum of the size-fractionated chlorophyll) and particulate organic carbon (Stramska and Stramski, 2005). Although these satellite data were all derived using empirical or semi-analytical algorithms, they have demonstrated considerable success in their agreement with in situ data. The uncertainty associated with these size-differentiated chlorophyll and POC concentrations has been estimated to be $35 \%$ (Pan et al., 2010; Stramska and Stramski, 2005).

Satellite-derived size-fractionated chlorophyll consists of three types of size-fractionated chlorophyll: large chlorophyll (ChlL), medium chlorophyll (ChlM) and small chlorophyll (ChlS), representing chlorophyll produced by microphytoplankton, nano-phytoplankton and pico-phytoplankton, respectively. When comparing the models with two phytoplankton components to these satellite data, the chlorophyll attributed to the large phytoplankton component was compared to ChlL, and the chlorophyll attributed to the small phytoplankton component was compared to the sum of ChlS + ChlM. When comparing the model with one phytoplankton component to these satellite data, the modeled chlorophyll was compared to the sum of all three types of chlorophyll.

\subsection{Data assimilation framework}

The specifics of the optimization implementation are well documented in Xiao and Friedrichs (2014), and thus only a brief description of the key properties of the variational adjoint data assimilative framework is provided here. 
Table 2. Number of observations (N), mean and standard deviation $(\sigma)$ of the satellite-derived chlorophyll concentrations (small, medium, large and total; $\left.\mathrm{mg} \mathrm{Chl} \mathrm{m}^{-3}\right)$ and $\mathrm{POC}$ data $\left(\mathrm{mg} \mathrm{C} \mathrm{m}^{-3}\right)$ at each site.

\begin{tabular}{|c|c|c|c|c|c|c|c|c|c|c|c|c|}
\hline & \multicolumn{9}{|c|}{ Chlorophyll } & \multirow{2}{*}{\multicolumn{3}{|c|}{ POC }} \\
\hline & & \multicolumn{2}{|c|}{ ChlS } & \multicolumn{2}{|c|}{ ChlM } & \multicolumn{2}{|c|}{ ChlL } & \multicolumn{2}{|c|}{ Total Chl } & & & \\
\hline & $\mathrm{N}$ & mean & $\sigma$ & mean & $\sigma$ & mean & $\sigma$ & mean & $\sigma$ & $\mathrm{N}$ & mean & $\sigma$ \\
\hline N1 & 108 & 0.03 & 0.02 & 0.38 & 0.30 & 0.22 & 0.23 & 0.63 & 0.52 & 119 & 134 & 48 \\
\hline $\mathrm{N} 2$ & 90 & 0.03 & 0.01 & 0.28 & 0.36 & 0.16 & 0.31 & 0.48 & 0.67 & 94 & 112 & 78 \\
\hline $\mathrm{S} 1$ & 122 & 0.04 & 0.02 & 0.40 & 0.33 & 0.24 & 0.25 & 0.68 & 0.57 & 124 & 140 & 54 \\
\hline S2 & 121 & 0.03 & 0.02 & 0.30 & 0.26 & 0.16 & 0.17 & 0.49 & 0.43 & 124 & 109 & 48 \\
\hline CV1 & 116 & 0.03 & 0.02 & 0.33 & 0.41 & 0.21 & 0.40 & 0.57 & 0.81 & 123 & 124 & 52 \\
\hline $\mathrm{CV} 2$ & 104 & 0.03 & 0.01 & 0.28 & 0.30 & 0.16 & 0.27 & 0.46 & 0.56 & 111 & 100 & 52 \\
\hline CV3 & 94 & 0.03 & 0.01 & 0.20 & 0.15 & 0.11 & 0.12 & 0.33 & 0.27 & 100 & 95 & 52 \\
\hline CV4 & 108 & 0.04 & 0.03 & 0.84 & 0.74 & 0.57 & 0.72 & 1.46 & 1.44 & 118 & 219 & 66 \\
\hline CV5 & 120 & 0.04 & 0.02 & 0.56 & 0.42 & 0.33 & 0.28 & 0.93 & 0.68 & 100 & 95 & 52 \\
\hline
\end{tabular}

The variational adjoint method is a nonlinear, weighted least-squares optimization method that minimizes the misfit between the model estimates and the observational data by optimizing a subset of model parameters (e.g., Lawson et al., 1995, 1996). The choice of parameters for optimization depends strongly on the data available for optimization. When size-differentiated chlorophyll and particulate organic carbon data are available for assimilation, Xiao and Friedrichs (2014) determined that successful assimilation results are obtained as long as data from multiple sites are assimilated, and the subset of parameters to be optimized includes maximum chlorophyll : carbon $(\mathrm{Chl}: \mathrm{C})$ ratios, maximum phytoplankton growth rates and zooplankton basal metabolism rates. Because each optimized parameter is size specific (that is, each phytoplankton size class has a distinct $\mathrm{Chl}: \mathrm{C}$ ratio and growth rate) and each zooplankton size class has a distinct basal metabolism rate (Table 1), the number of optimized parameters increases with increasing model complexity. For the five models tested here, 3, 5, 6, 7 and 8 parameters are optimized, respectively.

In this methodology the model-data misfit, otherwise known as the "cost function" $(J)$, is minimized, where

$J=\frac{1}{M} \sum_{k=1}^{K} \sum_{m=1}^{M} \frac{1}{N_{k m} \cdot \sigma_{k m}^{2}} \sum_{j=1}^{N_{k m}}\left(a_{j k m}-\hat{a}_{j k m}\right)^{2}$,

where $a$ represents the modeled equivalents to the observations ( $\hat{a}) ; M$ is the number of data types, for which $M=2,3$ or 4 depending on the number of $\mathrm{P}$ size classes resolved by the model; $K$ is the number of sites; $N_{k m}$ is the number of observations at site $k$ for data type $m$; and $\sigma_{k m}$ is the standard deviation of the $N_{k m}$ observations (Table 2). In this way, the cost function provides an estimate of the ratio between the model-data differences and the differences between the data and the mean of the data, i.e., $\sigma_{k m}^{2}$.

After the cost function is computed from an a priori forward model run, the adjoint code (Giering and Kaminski,
1998) computes the gradients of the cost function and passes the information to an optimization procedure (Gilbert and Lemaréchal, 1989), which determines how each optimized parameter value should be modified in order to reduce the magnitude of the cost function. The new parameter values are then used in another forward model run, the new cost function is computed, and the optimization procedure is repeated. These iterations continue until the specified convergence criterion is satisfied.

Following the recommendations of Xiao and Friedrichs (2014), both particulate organic carbon and sizedifferentiated chlorophyll were assimilated. Although this previous study found that POC estimates were not significantly improved as a result of the assimilation, the POC assimilation played a critical role in preventing significant deterioration of other state variables (zooplankton, detritus) that are included as components of POC. Thus the cost that was minimized by the optimization routine consists of the sum of these two components:

Size_cost $=$ SizeChl_cost + POC_cost ,

where SizeChl_cost represents that portion of the cost due to the model-data misfits of size-differentiated chlorophyll, and POC_cost represents the portion of the cost due to the POC model-data misfits. For the 1P model, SizeChl_cost is computed for total chlorophyll $(\mathrm{ChlS}+\mathrm{ChlM}+\mathrm{ChlL})$ and thus $M=2$ in Eq. (1) (i.e., one data type is total $\mathrm{ChlS}+\mathrm{ChlM}+\mathrm{ChlL}$ and one is POC.) For the $2 \mathrm{P}$ models, SizeChl_cost is computed as the sum of two separate components: ChlS + ChlM and ChlL. In this case $M=3$ (data types are ChlS + ChlM, ChlL and POC.) Finally, for the 3P models, SizeChl_cost includes misfits for ChlS, ChlM and ChlL separately, and four data types are assimilated $(M=4$ : ChlS, ChlM, ChlL and POC.)

As a result of the nonlinearities in the cost function formulation (Eq. 1), SizeChl_cost is not comparable across models with different numbers of phytoplankton variables, and thus 
Size_cost is not an appropriate metric for comparing the relative skill of all five models. Thus it is also critical to compute and compare the total cost (Total_cost) from the misfits in total chlorophyll and POC for the five models:

Total_cost $=$ TotChl_cost + POC_cost,

where TotChl_cost represents the model-data misfits in total chlorophyll concentration. (Note that Total_cost =Size_cost for the model with a single phytoplankton size class, since in this case the size-fractionated chlorophyll is identical to the total chlorophyll.) In this way, although for four of the five models Total_cost does not precisely correspond to the cost that is minimized through the optimization process, it provides a standard metric that can be used to rigorously compare the relative skill of all five ecosystem models.

\subsection{Model implementation and assimilation experiments}

The five ecosystem models were implemented in the framework described above at nine locations (Fig. 1) in the MidAtlantic Bight (MAB). Four of these sites were designated as "data assimilation" (DA) sites, since these are the locations at which data were assimilated. Two sites were selected on the inner shelf, and two were chosen just seaward of the shelf break, with two of the sites being located in the northern portion of the MAB and two in the southern portion. The remaining five sites were designated as "cross-validation" (CV) sites, since these were sites where the optimal parameters derived from assimilating data at the DA sites were independently tested. These sites were spread throughout the northern, central and southern MAB, with some sites located in shallow shelf waters, and other sites located off the shelf in deep (> $2000 \mathrm{~m}$ ) waters. Three experiments were conducted at these nine sites, and are described in more detail below.

- Experiment 1: each model was implemented in a forward model run at all nine sites, and a priori cost functions (both Size_cost and Total_cost) from these preassimilation simulations were computed.

- Experiment 2: POC data and size-fractionated chlorophyll data from the four DA sites were assimilated into each of the five models to determine a single best-fit set of parameter values for these four sites. The resulting cost functions (both Size_cost and Total_cost) were computed both at the four DA sites, as well as at the five CV sites.

- Experiment 3: to determine the robustness of the optimal parameters determined in experiment 2 and the sensitivity of these parameter values to uncertainties associated with the satellite-derived products, normally distributed random noise with a maximum amplitude of $20 \%$ was added to the size-fractionated chlorophyll and POC data from the four DA sites prior to assimilation.

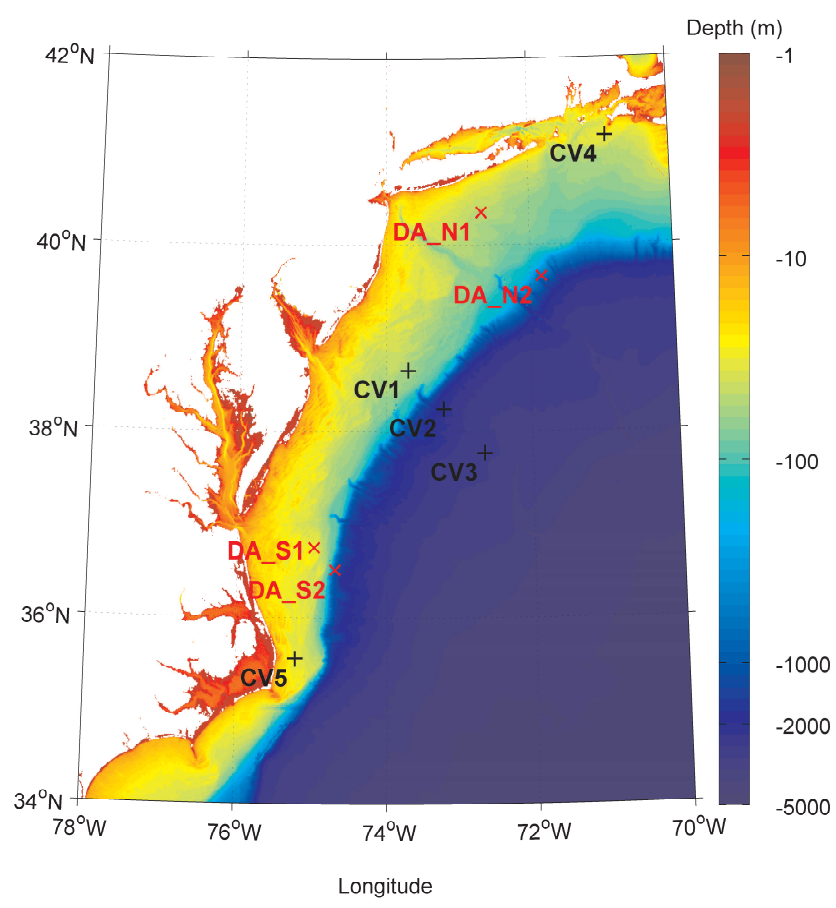

Figure 1. Locations of the nine study sites in the Mid-Atlantic Bight. The red crosses represent the four data assimilation (DA) sites, and the black pluses the five cross-validation (CV) sites.

The resulting optimal parameter values were compared to those determined in experiment 2. Cost functions for the four DA and five CV sites were computed as misfits between the simulations using these new optimal parameter values and the noisy data.

\section{Results}

\subsection{Experiment 1: a priori simulation}

All five a priori surface chlorophyll simulations from the five different models were comparable at most of the nine sites, in particular at the northern sites such as N1, N2, CV1 and CV2 (Fig. 2a). More variability between models was found at the southern sites and offshore sites. For example, the model estimates of the peak chlorophyll during the fall bloom ranged from $2 \mathrm{mg} \mathrm{Chl} \mathrm{m}^{-3}$ (the $2 \mathrm{P} 1 \mathrm{Z}$ model) to $>5 \mathrm{mg} \mathrm{Chl} \mathrm{m}^{-3}$ (the $3 \mathrm{P} 2 \mathrm{Z}$ model) at the CV5 site. The $1 \mathrm{P} 1 \mathrm{Z}$ model stood out from the other four models in that it tended to produce slightly higher chlorophyll concentrations at most of the sites, while it still gave similar estimates on the bloom timing to the other models (Fig. 2a).

In terms of size fractions (not shown), the simulations generated by the $2 \mathrm{P}$ and the $3 \mathrm{P}$ models also resembled one another at most sites. For example, ChlL concentrations remained low at all nine sites $(<10 \%$ of total chlorophyll) for most of the year except during the spring and fall blooms. For 
(a) A priori simulation

DA_N1
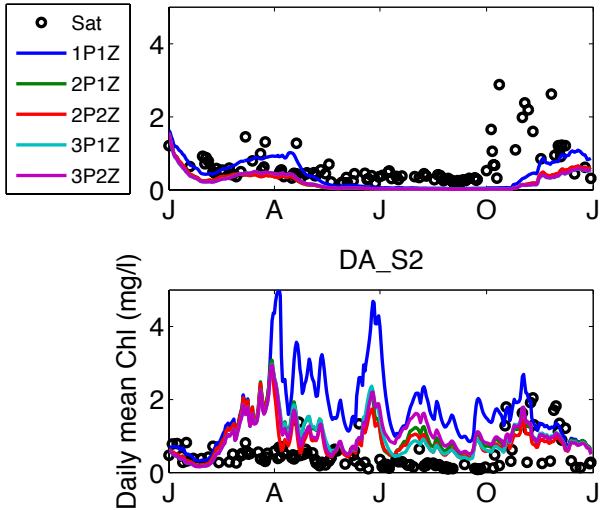

$\mathrm{CV} 3$

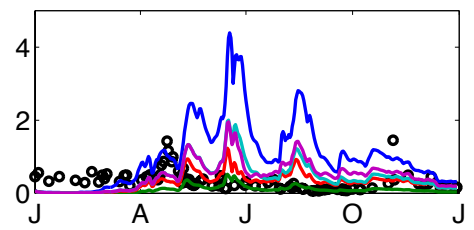

DA S1

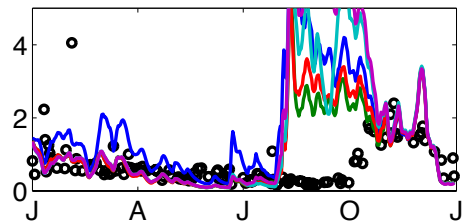

$\mathrm{CV} 2$

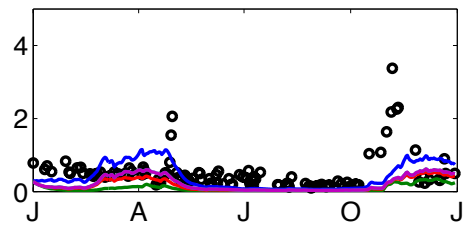

CV4

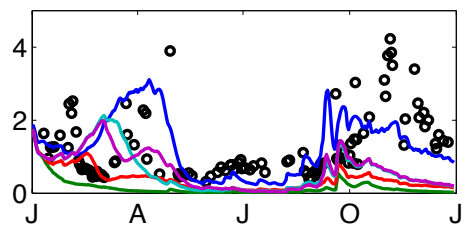

CV5

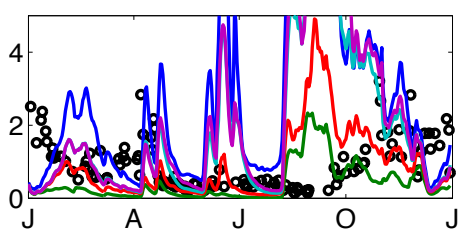

(b) A posteriori simulation
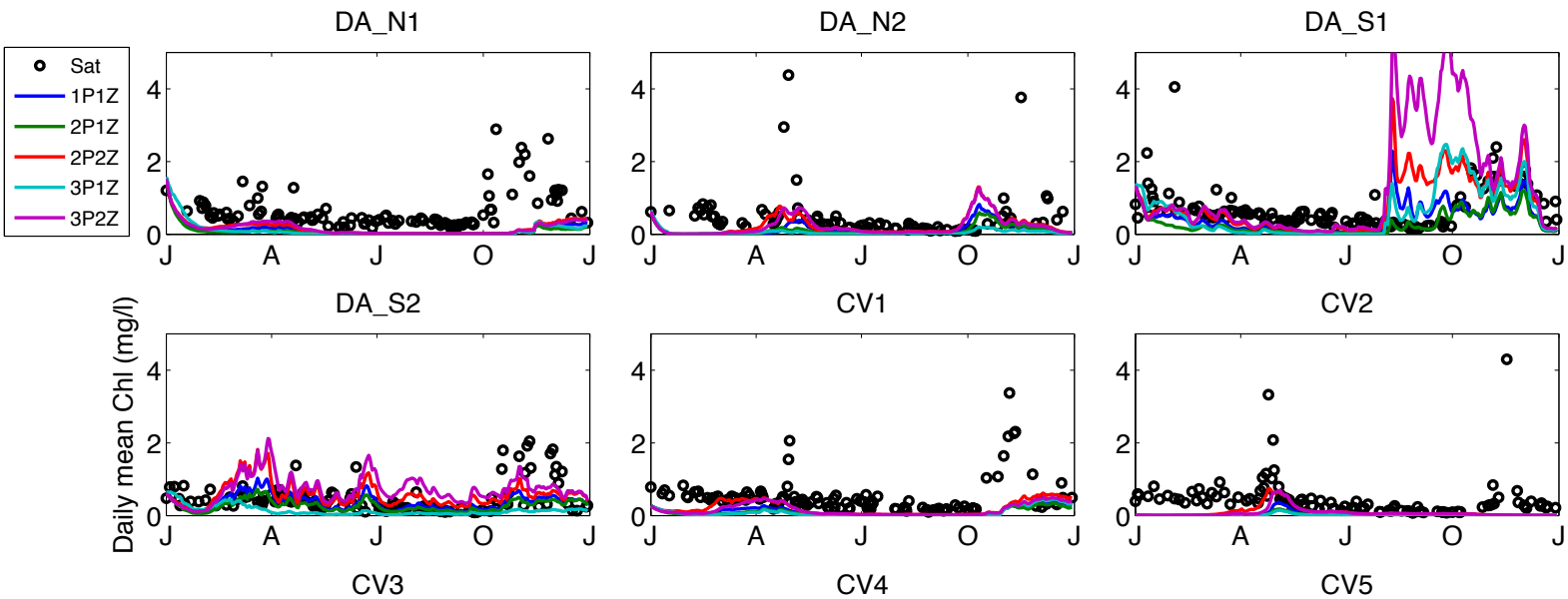

CV1

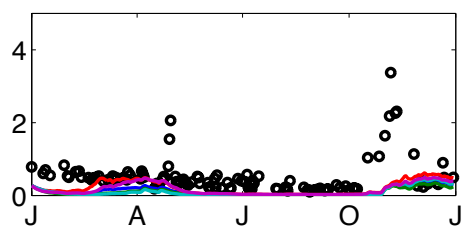

CV4
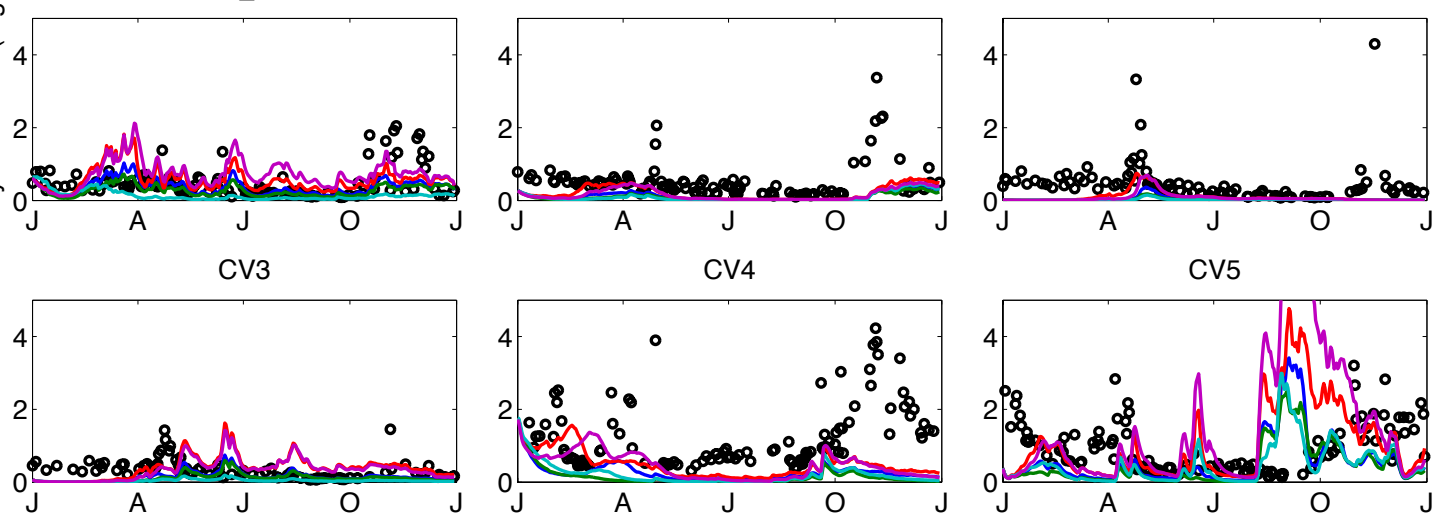

CV5

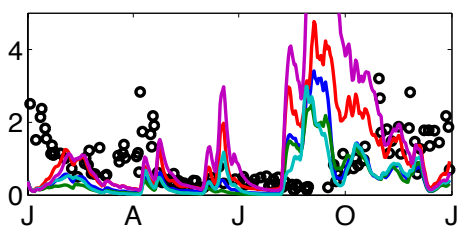

Figure 2. Time series of total surface chlorophyll from the satellite-derived data (open black circles) and the (a) a priori and (b) a posteriori simulations (lines) at the nine study sites for the five ecosystem models.

the 3P models, model estimates of ChlM were also considerably lower than ChlS throughout the year at all nine sites. For all $2 \mathrm{P}$ and $3 \mathrm{P}$ models, ChlS was the dominant chlorophyll component throughout most of the year.
Although all models failed to capture some key features of the surface chlorophyll distributions (Fig. 2a) such as bloom timing (e.g., at site DA_S1) and magnitude (e.g., at site DA_N2), in general, all five models fit the satellite-derived surface total chlorophyll and POC distributions similarly 
well. The general consistency in the five model simulations resulted in the a priori cost functions of the five models being relatively comparable. At both the DA sites (Table 3 ) and the CV sites (Table 4) the a priori Total_cost was highest for the simplest $1 \mathrm{P} 1 \mathrm{Z}$ model, primarily as a result of an overestimate of surface chlorophyll at the DA_S2 site and the offshore CV3 site (Fig. 2a). The 3P models performed only slightly better, as they significantly overestimated chlorophyll at the CV5 site near Cape Hatteras (Fig. 2a). In terms of reproducing the size fractionation data (Size_cost), the 2P models performed best, regardless of whether or not they included a second zooplankton component (Tables 3, 4). In terms of the 3P models, the model with the second zooplankton component produced slightly lower a priori Size_costs.

\subsection{Experiment 2: assimilation of satellite-derived data}

\subsubsection{Experiment 2 results at data assimilation (DA) sites}

The assimilation of size-differentiated chlorophyll and POC data at the four DA sites resulted in significant reductions in Size_cost (Table 3), indicating successful optimizations for all five models. Improvements in model-data misfit were most substantial at the two southern stations (DA_S1 and DA_S2) (Fig. 2b). As expected from the previous results of Xiao and Friedrichs (2014), this reduction in Size_cost was accomplished primarily through improvements in chlorophyll model-data fit (Fig. 3a and b). The assimilation particularly improved model-data misfit for the smallest size class of chlorophyll for all five models. The $2 \mathrm{Z}$ models also produced improved model-data fits for other size classes of chlorophyll, but this was not the case for the $1 \mathrm{Z}$ models.

Although Size_cost cannot be used to quantitatively compare the skill of all five models (see Sect. 2.3), it is still a useful metric for comparison of models with the same numbers of phytoplankton variables. Somewhat surprisingly, Size_cost was lower (and percent reduction in cost much greater) for models with only one zooplankton size class than for those with two zooplankton size classes. This effect was stronger for the more complex $3 \mathrm{P}$ models than for the $2 \mathrm{P}$ models (Table 3 ).

In order to compare models with different phytoplankton structures, Total_cost was computed to represent the modeldata misfits of total chlorophyll and POC (Table 3, Fig. 3c). After assimilation, Total_cost decreased for all models (mean decrease of $\sim 30 \%$ ), which was only slightly smaller than the analogous decrease of Size_cost (mean decrease of $\sim 40 \%$ ). The lowest a posteriori costs were found with the simplest $1 \mathrm{P}$ and $2 \mathrm{P}$ models, and the highest cost was obtained using the most complex 3P2Z model. The decrease in cost function was attained almost entirely through the decrease in chlorophyll cost (mean decrease of $\sim 55 \%$ ).

Optimal parameters generated by the five models were all well constrained (Fig. 4a). Out of the 29 optimized parame- (a)
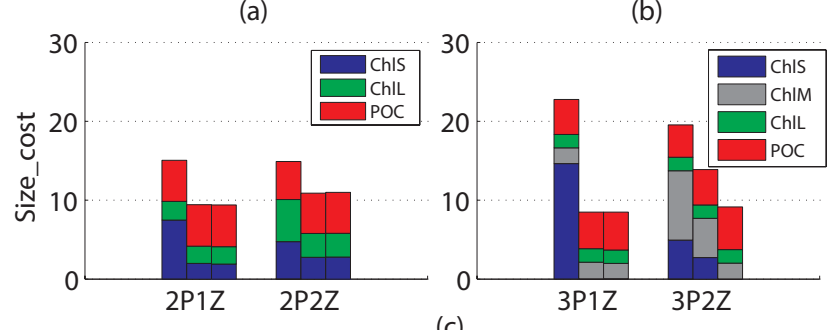

(c)

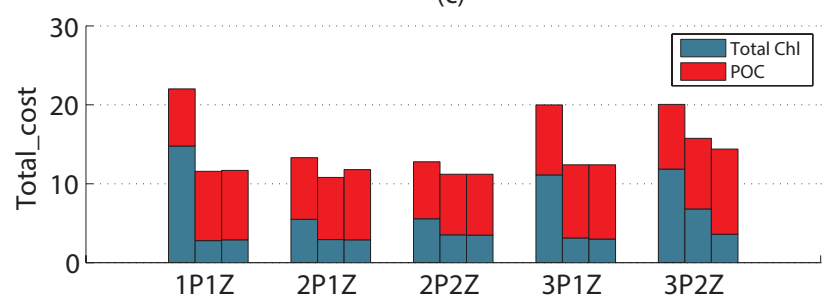

Figure 3. Cost functions at the four DA sites: (a) Size_cost for the 2P models, (b) Size_cost for the 3P models and (c) Total_cost for all five models. The three bars (from left to right) for each model represent the costs obtained for experiment 1 (a priori cost), experiment 2 (a posteriori cost) and experiment 3 (a posteriori case with noise), respectively. Colors represent the various components (total chlorophyll, size-fractionated chlorophyll and POC) of these costs.

ters for the 5 models, only 7 of these represented a change of greater than $50 \%$. Both $2 \mathrm{Z}$ models showed only minor changes in parameter values, whereas the three $1 \mathrm{Z}$ models all had at least one parameter that changed by more than $50 \%$. The large changes in parameter values for these $1 \mathrm{Z}$ models are consistent with the largest reductions in costs for these models, as discussed above. However, the 2P2Z model fit the total chlorophyll data (Total_cost $=11.2$ ) nearly as well as the $2 \mathrm{P} 1 \mathrm{Z}$ model (Total_cost $=10.8$ ), despite much smaller changes to the a priori parameter values. Specifically, the superior fit of the $2 \mathrm{P} 1 \mathrm{Z}$ model was obtained only when the maximum $\mathrm{Chl}: \mathrm{C}$ ratio for micro-phytoplankton was unrealistically reduced by an order of magnitude.

Among the three types of optimized parameters, the maximum phytoplankton growth rate was adjusted the least by the optimization, suggesting that these parameters were initialized near their optimal values. Greater variations in optimal values were found with the other parameters, without any clear patterns forming as a function of model structure.

\subsubsection{Experiment 2 results at cross-validation $(\mathrm{CV})$ sites}

By definition, the data assimilation improved model skill at the DA sites (Table 3) where the data were assimilated; however a more robust test of the optimization is to evaluate the optimized models against data at the CV sites (Table 4) where no data were assimilated (Gregg et al., 2009). When the optimal parameter sets obtained from assimilating the data at the DA sites were applied to another five nearby sites (CV sites in Fig. 1), Size_cost was reduced for 
Table 3. Cost functions (Size_cost and Total_cost) computed at the four DA sites in experiment 2, using initial parameter values (i.e., a priori cost) and optimal parameter values obtained from the assimilation of satellite-derived size-fractionated chlorophyll and POC data at the four DA sites (i.e., a posteriori cost).

\begin{tabular}{rrrrrrrrr}
\hline \multirow{3}{*}{ Model } & \multicolumn{3}{c}{ Size_cost } & & \multicolumn{3}{c}{ Total_cost } \\
\cline { 2 - 4 } \cline { 6 - 8 } & $\begin{array}{r}\text { a priori } \\
\text { cost }\end{array}$ & $\begin{array}{r}\text { a posteriori } \\
\text { cost }\end{array}$ & $\begin{array}{r}\% \\
\text { change }\end{array}$ & & $\begin{array}{r}\text { a priori } \\
\text { cost }\end{array}$ & $\begin{array}{r}\text { a posteriori } \\
\text { cost }\end{array}$ & $\begin{array}{r}\% \\
\text { change }\end{array}$ \\
\hline 1P1Z & 22.0 & 11.6 & $-47 \%$ & & 22.0 & 11.6 & $-47 \%$ \\
$2 \mathrm{P} 1 \mathrm{Z}$ & 15.1 & 9.4 & $-37 \%$ & & 13.3 & 10.8 & $-19 \%$ \\
$2 \mathrm{P} 2 \mathrm{Z}$ & 14.9 & 10.9 & $-26 \%$ & & 12.8 & 11.2 & $-12 \%$ \\
$3 \mathrm{P} 1 \mathrm{Z}$ & 22.8 & 8.5 & $-63 \%$ & & 20.0 & 12.4 & $-38 \%$ \\
$3 \mathrm{P} 2 \mathrm{Z}$ & 19.5 & 13.9 & $-29 \%$ & & 20.1 & 15.8 & $-21 \%$ \\
\hline
\end{tabular}

Table 4. Cost functions (Size_cost and Total_cost) computed at the five independent CV sites in experiment 2, using initial parameter values (i.e., a priori cost) and optimal parameter values obtained from the assimilation of satellite-derived size-fractionated chlorophyll and POC data at the four DA sites (i.e., a posteriori cost).

\begin{tabular}{|c|c|c|c|c|c|c|}
\hline \multirow{2}{*}{ Model } & \multicolumn{3}{|c|}{ Size_cost } & \multicolumn{3}{|c|}{ Total_cost } \\
\hline & $\begin{array}{r}\text { a priori } \\
\text { cost }\end{array}$ & $\begin{array}{r}\text { a posteriori } \\
\text { cost }\end{array}$ & $\begin{array}{r}\% \\
\text { change }\end{array}$ & $\begin{array}{r}\text { a priori } \\
\text { cost }\end{array}$ & $\begin{array}{r}\text { a posteriori } \\
\text { cost }\end{array}$ & $\begin{array}{r}\% \\
\text { change }\end{array}$ \\
\hline 1P1Z & 36.4 & 21.0 & $-42 \%$ & 36.4 & 21.0 & $-42 \%$ \\
\hline 2P1Z & 17.2 & 17.2 & $0 \%$ & 18.9 & 21.3 & $+13 \%$ \\
\hline $2 \mathrm{P} 2 \mathrm{Z}$ & 17.3 & 14.0 & $-19 \%$ & 27.2 & 15.3 & $-43 \%$ \\
\hline $3 \mathrm{P} 1 \mathrm{Z}$ & 23.7 & 13.5 & $-43 \%$ & 32.7 & 22.2 & $-32 \%$ \\
\hline $3 \mathrm{P} 2 \mathrm{Z}$ & 19.4 & 16.0 & $-17 \%$ & 34.7 & 20.4 & $-41 \%$ \\
\hline
\end{tabular}

all models except the 2P1Z model (Table 4, Fig. 5a, b). The greatest reductions in Size_cost at the CV sites occurred for the $3 \mathrm{P} 1 \mathrm{Z}$ and $1 \mathrm{P} 1 \mathrm{Z}$ models $(\sim 40 \%)$, which was equivalent to the reductions in Size_cost generated by these models at the DA sites. Significant, but smaller, reductions also occurred for the $2 \mathrm{P} 2 \mathrm{Z}$ and $3 \mathrm{P} 2 \mathrm{Z}$ models $(\sim 20 \%$, Table 4$)$. All five models showed an increase in the POC cost; however the improvement in model-data fit for size-fractionated chlorophyll, particularly for the smallest chlorophyll size class, more than compensated for the deterioration in POC modeldata misfit in all cases except for the $2 \mathrm{P} 1 \mathrm{Z}$ model (Fig. $5 \mathrm{a}$ and b).

Applying the optimal parameters from the DA sites to simulations at the CV sites also generated significant improvements in the total chlorophyll cost for each of the five models (Fig. 5c). This decrease in total chlorophyll cost was again substantially larger than the increase in POC cost for all models except the 2P1Z model, and thus the overall Total_cost also decreased for four of the five models (Table 4). The lack of improvement for the $2 \mathrm{P} 1 \mathrm{Z}$ model is at least partially due to the fact that using the a priori parameter values with the $2 \mathrm{P} 1 \mathrm{Z}$ model generated an a priori simulation that fit the data at the five CV sites very well (Fig. 5c). In fact the a priori Total_cost for the 2P1Z model was lower than the a posteriori Total_cost of the 1P and 3P models (Table 4, Fig. 5c). Overall, the intermediately complex $2 \mathrm{P} 2 \mathrm{Z}$ model produced the lowest Total_cost when using the parameters optimized for the DA sites at the $\mathrm{CV}$ sites.

\subsection{Experiment 3: assimilation of perturbed data}

\subsubsection{Experiment 3 results at data assimilation (DA) sites}

The a priori costs for experiment 3 were computed as the difference between the a priori simulations and the noisy data, and were only very slightly different $(<1 \%)$ from the a priori costs for experiment 2 , which were computed as the difference between the a priori simulations and the actual data.

When the noisy data were assimilated into the models at the four DA sites in experiment 3, the optimization process generated very similar parameters to those generated in experiment 2 for the 1P, $2 \mathrm{P}$ and $3 \mathrm{P} 1 \mathrm{Z}$ models (Fig. 4). Thus the addition of random noise did not significantly affect the optimization process for these simpler models, and as a result the a posteriori Size_costs resulting from the assimilation of the noisy data were almost identical to those generated by assimilating the actual data (Fig. 3).

In contrast, the optimal parameters generated in experiment 3 for the most complex 3P2Z model were significantly different from those in experiment 2 (Fig. 4b). For example, the optimal value for the maximum $\mathrm{Chl}$ : C ratio for pico-phytoplankton in the $3 \mathrm{P} 2 \mathrm{Z}$ model was 
(a) Expt. 2

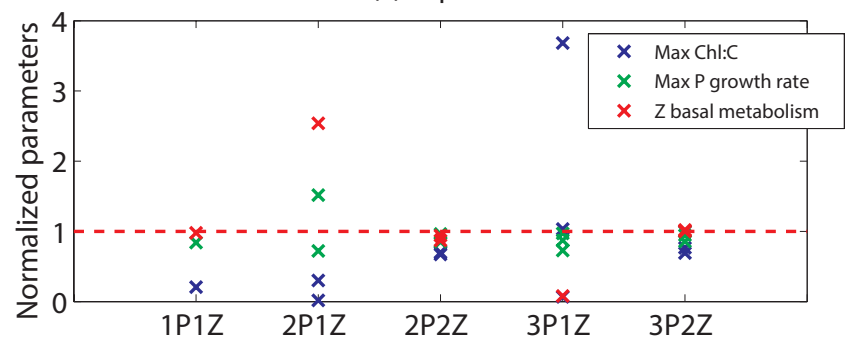

(b) Expt. 3

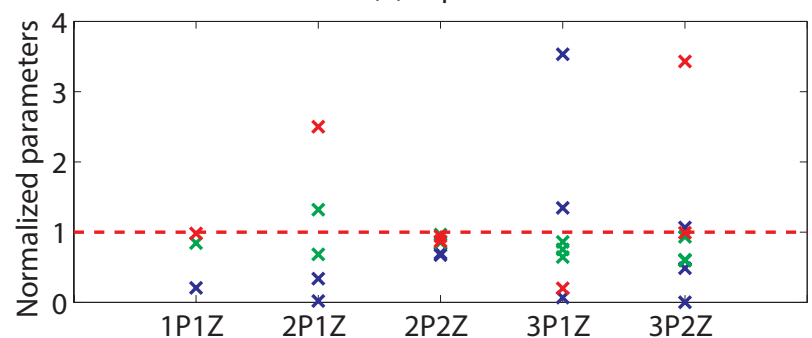

Figure 4. Optimized parameter values normalized to a priori values obtained (a) by assimilating POC and size-fractionated data at the four DA sites (experiment 2), and (b) by assimilating satellite data to which $20 \%$ random noise has been added (experiment 3 ).

(a)
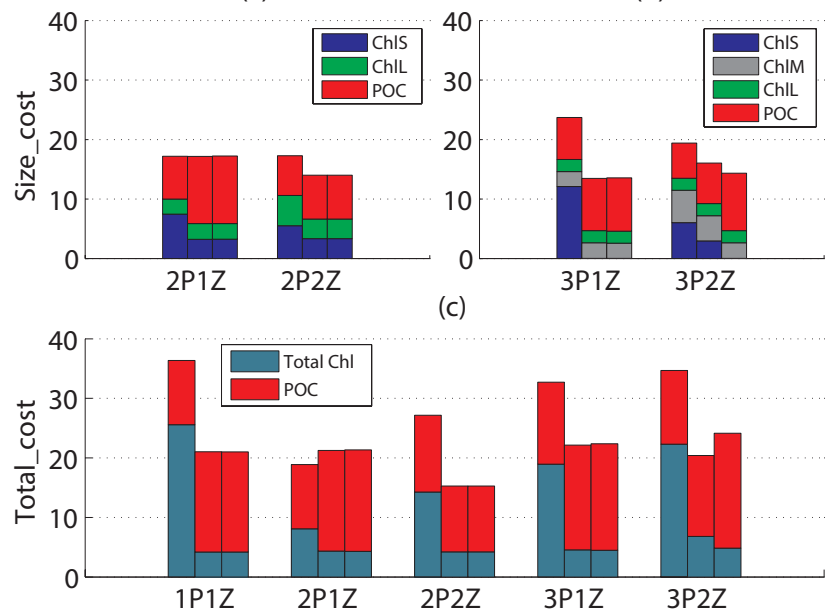

Figure 5. Cost functions at the five CV sites: (a) Size_cost for the 2P models, (b) Size_cost for the 3P models and (c) Total_cost for all five models. The three bars (from left to right) for each model represent the costs obtained for experiment 1 (a priori cost), experiment 2 (a posteriori cost) and experiment 3 (a posteriori case with noise), respectively. Colors represent the various components (total chlorophyll, size-fractionated chlorophyll and POC) of these costs.

$6.1 \times 10^{-13} \mathrm{mg} \mathrm{Chl} \mathrm{mg} \mathrm{C} \mathrm{C}^{-1}$ compared to a value of 0.023 generated when assimilating the actual satellite-derived data. As a result, this new set of optimal parameter values (Fig. 4b) resulted in a significantly different Size_cost $(\sim 35 \%$ decrease). This decrease in the 3P2Z Size_cost was caused by a substantial reduction in the cost components of ChlS and ChlM, whereas the contribution of ChlL and POC remained nearly unchanged (Fig. 3b).

\subsubsection{Experiment 3 results at cross-validation (CV) sites}

The costs at the $\mathrm{CV}$ sites for the $1 \mathrm{P}, 2 \mathrm{P}$ and $3 \mathrm{P} 1 \mathrm{Z}$ models were nearly identical for experiments 2 and 3 (Fig. 5). This was true despite some significant changes in the optimized parameter values for the 3P1Z model (Fig. 4): for example, the zooplankton basal metabolism rate was twice as high in experiment 3 compared to experiment 2 . As was the case at the DA sites, the $3 \mathrm{P} 2 \mathrm{Z}$ a posteriori costs were much more sensitive to the noise added to the data prior to assimilation. Although the a posteriori 3P2Z Size_cost decreased for the ChlS and ChlM components, the a posteriori Total_cost increased due to a significant deterioration in the model-data fit for POC.

In summary, the addition of noise to the assimilated data had almost no effect on the cost functions for the simpler models, but significantly affected the costs of the most complex (3P2Z) model. Although the 3P2Z model showed improvement in model-data misfit at the DA sites with the addition of noise prior to assimilation, it was attained at the expense of unreasonable optimized parameter values and an increase in the Total_cost at the independent cross-validation sites.

\section{Discussion and conclusions}

In this study, five lower-trophic-level ecosystem models with varying food web complexities were rigorously compared in order to determine how the number of phytoplankton and zooplankton compartments affects the ability of a lowertrophic-level model to reproduce observed patterns in surface chlorophyll and particulate organic carbon. All five models were embedded in a 1-D assimilative model framework with identical physics and biogeochemical formulations, and thus the differences in the model simulations were only a result of variations in the complexity of the planktonic food web structure.

As expected based on previous studies assimilating satellite-derived data fields into marine ecosystem models (Fan and Lv, 2009; Friedrichs, 2002; Garcia-Gorriz et al., 2003; Hemmings et al., 2004; Tjiputra et al., 2007), all models tested here showed improvement in model skill after the assimilation of the satellite-derived fields and resulting optimization of the plankton-related parameters. Whereas prior to assimilation the five models varied somewhat in their ability to fit the satellite-derived data fields, after assimilation the models produced total chlorophyll and POC fields at the assimilation sites that matched the satellite data nearly equally well. However, even after assimilation, all five models still had difficulty reproducing the seasonal cycle of this system. 
This is most likely due to issues related to the physical fields obtained from the 3-D simulation used to force these models.

Interestingly, the a posteriori parameters optimized for these five models were very different for the different models. In particular, the models with a single zooplankton size class were only able to reproduce the assimilated data using extremely low zooplankton basal metabolism rates, or extremely low maximum $\mathrm{Chl}: \mathrm{C}$ ratios, whereas the models with two zooplankton size classes were able to reproduce the POC and chlorophyll observations using realistic rates and ratios. Ultimately, the parameters optimized for the twophytoplankton, two-zooplankton (2P2Z) model were most similar to our best-guess a priori initial parameter values.

The improvements in model skill for all five models were not limited to the four specific sites where the data were assimilated. Rather, a cross-validation analysis demonstrated that the parameters optimized for these four sites within the MAB improved the simulations at a number of other sites throughout the region, giving us confidence in the portability of these optimized parameter values and optimism for the potential success of using these parameters in a threedimensional simulation of the US eastern continental shelf (McDonald et al., 2012). Although almost all models showed some degree of improvement at these other MAB sites, once again the model characterized by intermediate complexity (i.e., 2P2Z) performed best. The other models were able to fit the data at the assimilation sites equally as well as the 2P2Z model; however, they typically did so by using unrealistic parameter values which were not portable to other areas of the MAB.

Intriguing results were also obtained when random noise was added to the satellite-derived data prior to assimilation. The addition of the noise perturbation had almost no effect on the values of the optimized parameters for the simplest four models, suggesting that the optimization process was robust for these models, even when significant noise was present in the assimilated data. However, when these perturbed data were assimilated into the most complex model (the 3P2Z model), substantially different optimal parameter values were obtained. For certain parameters (e.g., the maximum $\mathrm{Chl}: \mathrm{C}$ ratio for pico-phytoplankton), the difference between the optimized parameter values obtained by assimilating the actual data versus those obtained by assimilating the noisy data was more than 10 orders of magnitude. Although the new parameter values obtained by assimilating the noisy data improved the model-data fit at the specific sites where the data were assimilated, the unrealistic parameter values deteriorated the model performance at other sites within the MAB. In essence, unlike the simpler models, the most complex model had enough flexibility that it was actually able to fit the additional noise artificially added to the data. Although this "over-tuning" actually improved the model-data fit at the sites where the noisy data were assimilated, this is a dangerous outcome, as the model-data fit was degraded at other locations within the MAB where data were not available for assimilation.

This over-tuning issue for complex models has been alluded to in previous studies. For example, Friedrichs et al. (2006) assimilated data during three seasons of the year, and cross-validated the resulting optimal parameters against the data in the remaining season. Their cross-validation experiments showed that complex models with too many unconstrained parameters might be able to fit the assimilated data extremely well (the more free parameters, the better the fit to the assimilated data), yet these complex models would have poor predictive ability (the more free parameters, the worse the fit to independent, unassimilated data).

Another difficulty with complex models is that they are usually governed by such a large number of parameters (the number of parameters that must be specified in a given ecosystem model generally increases by as much as the square of the number of state variables; Denman, 2003), that it is very difficult to identify the best-fit set of parameters. When hand-tuning such models, there are simply too many different parameters to adequately test all parameter combinations. When applying an automated parameter optimization method such as the variational adjoint method to complex models with multiple unconstrained parameters, the cost function has a tendency to get stuck in suboptimal "local minima", and as a result the absolute global cost function minimum and the true "best-fit" set of parameters can potentially never be identified. In fact, this is exactly what occurred in the present study for the most complex $3 \mathrm{P} 2 \mathrm{Z}$ model. The a posteriori cost function was highest for this model, despite the presumably increased flexibility that this model had to fit the data, because the cost function became stuck in a local minimum. However, when artificial noise was added to the data prior to assimilation, an alternate local cost function minimum was identified, which, somewhat surprisingly, was smaller than the one identified when the true data were assimilated. The problem of complex models becoming stuck in local cost function minima has also been discussed by other authors. For example, Ward et al. (2010) demonstrated that when too many unconstrained parameters were optimized, the cost function often became trapped in a local minimum; however, reducing the number of optimized parameters partially eliminated this problem.

Our conclusion that an intermediate complexity model is the most ideally suited for regional ecosystem studies is consistent with results from earlier studies using other types of models without the formal parameter optimization techniques employed here. For example, an early study by Costanza and Sklar (1985) rated 87 models in wetland and shallow water bodies in terms of 3 indices: articulation (the complexity of the model), accuracy (goodness of fit of the model to the data) and effectiveness (trade-off between complexity and accuracy). They concluded that although the accuracy seemed to increase with articulation, the maximum effectiveness was found at an intermediate level of 
complexity. Fulton et al. (2003) found a similar humped relationship between model complexity and performance when examining end-to-end (nutrient to fisheries) models, demonstrating that the best performance was produced by the model with intermediate complexity. Another model comparison study was conducted by Raick et al. (2006), in which three simplified pelagic ecosystem models with 16,9 and 4 state variables, respectively, were assessed according to their ability to reproduce simulations from performance of a complex model with 19 state variables. The study found that although the simplest model (4 state variables) was able to capture the key features demonstrated by the complex model, the model with intermediate complexity ( 9 state variables) most closely reproduced the output from the full 19 state-variable model.
In summary, the study presented here provides additional evidence that lower-trophic-level food web models of intermediate complexity (e.g., containing two phytoplankton and two zooplankton compartments) are most likely to be able to provide best estimates of chlorophyll and carbon concentrations on regional scales such as the US eastern continental shelf. Simple models with only a single zooplankton size class may be able to reproduce observed data fields, but typically can only do so using unrealistic parameters that are not portable throughout the region. However, more complex models have difficulty finding cost minima and have issues with over-tuning and artificially fitting data noise, making them potentially unsuitable for extrapolating to locations and times where/when data may not be available for assimilation. 


\section{Appendix A: Model equations}

The equations for each of the five models are included below for reference. State variables are defined as follows: $\mathrm{P}$, total phytoplankton; Z, total zooplankton; SD, small detritus; LD, large detritus; NO3, nitrate; NH4, ammonium; Chl, chlorophyll; SP, small phytoplankton; MP, medium phytoplankton; LP, large phytoplankton; SZ, small zooplankton; LZ, large zooplankton; SChl, small chlorophyll; MChl, medium chlorophyll; and LChl, large chlorophyll. Parameter values, except for those specifically noted in text and Table 1, are provided in Fennel et al. (2006, 2008). As an example, generic functional formulations are listed below for SP and SZ, but analogous formulations hold for the medium (MP, $\mathrm{MZ})$, large (LP, LZ) and single (P, Z) size classes.

Functional formulations (example for SP, SZ)

$$
\begin{aligned}
& g_{\mathrm{SP} \_\mathrm{SZ}}=g_{\text {max } \_ \text {SP_SZ }} \frac{\mathrm{SP}^{2}}{k_{\mathrm{P}}+\mathrm{SP}^{2}} \\
& \rho_{\mathrm{SChl}}=\frac{\theta_{\mathrm{max}_{\_} \mathrm{SP}} \mu_{\mathrm{SP}} \cdot \mathrm{SP}}{\alpha I \cdot \mathrm{SChl}} \\
& f_{\mathrm{SP}}(I)=\frac{\alpha I}{\sqrt{\mu_{\mathrm{max} \_\mathrm{SP}}^{2}+\alpha^{2} I^{2}}} \\
& L_{\mathrm{NO} 3 \_\mathrm{SP}}=\left(\frac{\mathrm{NO} 3}{k_{\mathrm{NO} 3 \_\mathrm{SP}}+\mathrm{NO} 3}\right)\left(1+\frac{\mathrm{NH} 4}{k_{\mathrm{NH} 4}}\right)^{-1} \\
& L_{\mathrm{NH} 4}=\frac{\mathrm{NH} 4}{k_{\mathrm{NH} 4}+\mathrm{NH} 4} \\
& \mu_{\mathrm{SP}}=\mu_{\mathrm{max} \_\mathrm{SP}} f_{\mathrm{SP}}(I)\left(L_{\mathrm{NO} 3} \_\mathrm{SP}+L_{\mathrm{NH} 4}\right) \\
& \mu_{\mathrm{SP} \_\mathrm{NO} 3}=\mu_{\mathrm{max} \_\mathrm{SP}} f_{\mathrm{SP}}(I) L_{\mathrm{NO} 3 \_\mathrm{SP}} \\
& \mu_{\mathrm{SP} \_\mathrm{NH} 4}=\mu_{\text {max } \_\mathrm{SP}} f_{\mathrm{SP}}(I) L_{\mathrm{NH} 4}
\end{aligned}
$$

\section{P1Z (includes $P, Z$ )}

$$
\begin{aligned}
\frac{\partial \mathrm{P}}{\partial t} & =\mu_{\mathrm{P}} \mathrm{P}-g_{\mathrm{P} \_\mathrm{Z}} \mathrm{Z}-m_{\mathrm{P}} \mathrm{P}-\tau(\mathrm{SD}+\mathrm{P}) \mathrm{P}-w_{\mathrm{P}} \frac{\partial \mathrm{P}}{\mathrm{d} z} \\
\frac{\partial \mathrm{Z}}{\partial t} & =g_{\mathrm{P} \_\mathrm{Z}} \beta \mathrm{Z}-l_{\mathrm{BM} \_\mathrm{Z}} \mathrm{Z}-l_{\mathrm{E}} \frac{\mathrm{P}^{2}}{k_{\mathrm{P}}+\mathrm{P}^{2}} \beta \mathrm{Z}-m_{\mathrm{Z}} \mathrm{Z}^{2} \\
\frac{\partial \mathrm{SD}}{\partial t} & =g_{\mathrm{P} \_\mathrm{Z}}(1-\beta) \mathrm{Z}+m_{\mathrm{Z}} \mathrm{Z}^{2}+m_{\mathrm{P}} \mathrm{P}-\tau(\mathrm{SD}+\mathrm{P}) \mathrm{SD} \\
& -r_{\mathrm{SD}} \mathrm{SD}-w_{\mathrm{SD}} \frac{\partial \mathrm{SD}}{\partial z} \\
\frac{\partial \mathrm{LD}}{\partial t} & =\tau(\mathrm{SD}+\mathrm{P})^{2}-r_{\mathrm{LD}} \mathrm{LD}-w_{\mathrm{LD}} \frac{\partial \mathrm{LD}}{\partial z} \\
\frac{\partial \mathrm{NO} 3}{\partial t} & =-\mu_{\mathrm{P} \_\mathrm{NO} 3} \mathrm{P}+n \mathrm{NH} 4 \\
\frac{\partial \mathrm{NH} 4}{\partial t} & =-\mu_{\mathrm{P} \_\mathrm{NH} 4} \mathrm{P}-n \mathrm{NH} 4+l_{\mathrm{BM}} \mathrm{ZZ}+l_{\mathrm{E}} \frac{\mathrm{P}^{2}}{k_{\mathrm{P}}+\mathrm{P}^{2}} \beta \mathrm{Z} \\
& +r_{\mathrm{SD}} \mathrm{SD}+r_{\mathrm{LD}} \mathrm{LD} \\
\frac{\partial \mathrm{Chl}}{\partial t} & =\rho_{\mathrm{Ch} l} \mu_{\mathrm{P}} \mathrm{Chl}-g_{\mathrm{P} \_\mathrm{Z}} \frac{\mathrm{Chl}}{\mathrm{P}}-m_{\mathrm{P}} \mathrm{Chl}-\tau(\mathrm{SD}+\mathrm{P}) \mathrm{Chl}
\end{aligned}
$$

2P1Z (includes SP, LP, Z)

$$
\begin{aligned}
& \frac{\partial \mathrm{SP}}{\partial t}=\mu_{\mathrm{SP}} \mathrm{SP}-g_{\mathrm{SP} \_\mathrm{Z}}-m_{\mathrm{P}} \mathrm{SP}-\tau(\mathrm{SD}+\mathrm{P}) \mathrm{SP}-w_{\mathrm{SP}} \frac{\partial \mathrm{SP}}{\mathrm{d} z} \\
& \frac{\partial \mathrm{LP}}{\partial t}=\mu_{\mathrm{LP}} \mathrm{LP}-g_{\mathrm{LP}} \mathrm{Z}_{\mathrm{Z}}-m_{\mathrm{P}} \mathrm{LP}-\tau(\mathrm{SD}+\mathrm{P}) \mathrm{LP}-w_{\mathrm{LP}} \frac{\partial \mathrm{LP}}{\mathrm{d} z} \\
& \frac{\partial \mathrm{Z}}{\partial t}=\left(g_{\mathrm{SP} \_\mathrm{Z}}+g_{\mathrm{LP} \_\mathrm{Z}}\right) \beta \mathrm{Z}-l_{\mathrm{BM} \_\mathrm{Z}} \mathrm{Z}-l_{\mathrm{E}} \frac{\mathrm{P}^{2}}{k_{\mathrm{P}}+\mathrm{P}^{2}} \beta \mathrm{Z}-m_{\mathrm{Z}} \mathrm{Z}^{2} \\
& \frac{\partial \mathrm{SD}}{\partial t}=\left(g_{\mathrm{SP}} \mathrm{Z}+g_{\mathrm{LP} \_} \mathrm{Z}\right)(1-\beta) \mathrm{Z}+m_{\mathrm{Z}} \mathrm{Z}^{2}+m_{\mathrm{P}} \mathrm{P} \\
& -\tau(\mathrm{SD}+\mathrm{P}) \mathrm{SD}-r_{\mathrm{SD}} \mathrm{SD}-w_{\mathrm{SD}} \frac{\partial \mathrm{SD}}{\partial z} \\
& \frac{\partial \mathrm{LD}}{\partial t}=\tau(\mathrm{SD}+\mathrm{P})^{2}-r_{\mathrm{LD}} \mathrm{LD}-w_{\mathrm{LD}} \frac{\partial \mathrm{LD}}{\partial z} \\
& \frac{\partial \mathrm{NO} 3}{\partial t}=-\mu_{\mathrm{SP} \_\mathrm{NO} 3} \mathrm{SP}-\mu_{\mathrm{LP} \_\mathrm{NO} 3} \mathrm{LP}+n \mathrm{NH} 4 \\
& \frac{\partial \mathrm{NH} 4}{\partial t}=-\mu_{\mathrm{SP} \_\mathrm{NH} 4} \mathrm{SP}-\mu_{\mathrm{LP} \_\mathrm{NH} 4} \mathrm{LP}-n \mathrm{NH} 4+l_{\mathrm{BM} \_} \mathrm{Z} \\
& +l_{\mathrm{E}} \frac{\mathrm{P}^{2}}{k_{\mathrm{P}}+\mathrm{P}^{2}} \beta \mathrm{Z}+r_{\mathrm{SD}} \mathrm{SD}+r_{\mathrm{LD}} \mathrm{LD} \\
& \frac{\partial \mathrm{SChl}}{\partial t}=\rho_{\mathrm{SChl}} \mu_{\mathrm{SP}} \mathrm{SChl}-g_{\mathrm{SP} \_\mathrm{Z}} \frac{\mathrm{SChl}}{S P}-m_{\mathrm{P}} \mathrm{SChl} \\
& -\tau(\mathrm{SD}+\mathrm{P}) \mathrm{SChl} \\
& \frac{\partial \mathrm{LChl}}{\partial t}=\rho_{\mathrm{LChl}} \mu_{\mathrm{LP}} \mathrm{LChl}-g_{\mathrm{LP} \_\mathrm{ZZ}} \frac{\mathrm{LChl}}{L P}-m_{\mathrm{P}} \mathrm{LChl} \\
& -\tau(\mathrm{SD}+\mathrm{P}) \mathrm{LChl}
\end{aligned}
$$

2P2Z (includes SP, LP, SZ, LZ)

$$
\begin{aligned}
& \frac{\partial \mathrm{SP}}{\partial t}=\mu_{\mathrm{SP}} \mathrm{SP}-g_{\mathrm{SP} \_\mathrm{SZ}} \mathrm{SZ}-g_{\mathrm{SP} \_\mathrm{LZ}} \mathrm{LZ}-m_{\mathrm{P}} \mathrm{SP} \\
& -\tau(\mathrm{SD}+\mathrm{P}) \mathrm{SP}-w_{\mathrm{SP}} \frac{\partial \mathrm{SP}}{\mathrm{d} z} \\
& \frac{\partial \mathrm{LP}}{\partial t}=\mu_{\mathrm{LP}} \mathrm{LP}-g_{\mathrm{LP} \_\mathrm{SZ}} \mathrm{SZ}-g_{\mathrm{LP} \_\mathrm{LZ}} \mathrm{LZ}-m_{\mathrm{P}} \mathrm{LP} \\
& -\tau(\mathrm{SD}+\mathrm{P}) \mathrm{LP}-w_{\mathrm{LP}} \frac{\partial \mathrm{LP}}{\mathrm{d} z} \\
& \frac{\partial \mathrm{SZ}}{\partial t}=\left(g_{\mathrm{SP} \_\mathrm{SZ}}+g_{\mathrm{LP} \_\mathrm{SZ}}\right) \beta_{\mathrm{SZ}}-l_{\mathrm{BM} \_\mathrm{SZ}} \mathrm{SZ}-l_{\mathrm{E}} \frac{\mathrm{P}^{2}}{k_{\mathrm{P}}+\mathrm{P}^{2}} \beta_{\mathrm{SZ}} \\
& -m_{\mathrm{Z}} \mathrm{SZ}^{2} \\
& \frac{\partial \mathrm{LZ}}{\partial t}=\left(g_{\mathrm{SP} \_\mathrm{LZ}}+g_{\mathrm{LP} \_\mathrm{LZ}}\right) \beta_{\mathrm{LZ}}-l_{\mathrm{BM} \_\mathrm{LZ}} \mathrm{LZ}-l_{\mathrm{E}} \frac{\mathrm{P}^{2}}{k_{\mathrm{P}}+\mathrm{P}^{2}} \beta_{\mathrm{LZ}} \\
& -m_{\mathrm{Z}} \mathrm{LZ} \mathrm{Z}^{2} \\
& \frac{\partial \mathrm{SD}}{\partial t}=\left(g_{\mathrm{SP} \_\mathrm{SZ}}+g_{\mathrm{LP} \_\mathrm{SZ}}\right)(1-\beta) \mathrm{SZ} \\
& +\left(g_{\mathrm{SP} \_\mathrm{LZ}}+g_{\mathrm{LP} \_\mathrm{LZ}}\right)(1-\beta) \mathrm{LZ}+m_{\mathrm{Z}} \mathrm{SZ}^{2}+m_{\mathrm{Z}} \mathrm{LZ}^{2} \\
& +m_{\mathrm{P}} \mathrm{P}-\tau(\mathrm{SD}+\mathrm{P}) \mathrm{SD}-r_{\mathrm{SD}} \mathrm{SD}-w_{\mathrm{SD}} \frac{\partial \mathrm{SD}}{\partial z}
\end{aligned}
$$




$$
\begin{aligned}
\frac{\partial \mathrm{LD}}{\partial t} & =\tau(\mathrm{SD}+\mathrm{P})^{2}-r_{\mathrm{LD}} \mathrm{LD}-w_{\mathrm{LD}} \frac{\partial \mathrm{LD}}{\partial z} \\
\frac{\partial \mathrm{NO} 3}{\partial t} & =-\mu_{\mathrm{SP} \_\mathrm{NO} 3} \mathrm{P}-\mu_{\mathrm{LP} \_\mathrm{NO} 3} \mathrm{P}+n \mathrm{NH} 4 \\
\frac{\partial \mathrm{NH} 4}{\partial t} & =-\mu_{\mathrm{SP} \_\mathrm{NH} 4} \mathrm{P}-\mu_{\mathrm{LP} \_\mathrm{NH} 4} \mathrm{P}-n \mathrm{NH} 4+l_{\mathrm{BM} \_\mathrm{SZ}} \mathrm{SZ} \\
& +l_{\mathrm{BM} \_\mathrm{LZ}} \mathrm{LZ}+l_{\mathrm{E}} \frac{\mathrm{P}^{2}}{k_{\mathrm{P}}+\mathrm{P}^{2}} \beta_{\mathrm{Z}}+r_{\mathrm{SD}} \mathrm{SD}+r_{\mathrm{LD}} \mathrm{LD} \\
\frac{\partial \mathrm{SChl}}{\partial t} & =\rho_{\mathrm{SChl}} \mu_{\mathrm{SP}} \mathrm{SChl}-g_{\mathrm{SP} \_\mathrm{SZ}} \mathrm{SZ} \frac{\mathrm{SChl}}{\mathrm{SP}}-g_{\mathrm{SP} \_\mathrm{LZ}} \mathrm{LZ} \frac{\mathrm{SChl}}{\mathrm{SP}} \\
& -m_{\mathrm{P} S C h l}-\tau(\mathrm{SD}+\mathrm{P}) \mathrm{SChl} \\
\frac{\partial \mathrm{LChl}}{\partial t} & =\rho_{\mathrm{LChl}} \mu_{\mathrm{LP}} \mathrm{LChl}-g_{\mathrm{LP} \_\mathrm{SZ}} \mathrm{SZ} \frac{\mathrm{LChl}}{\mathrm{LP}}-g_{\mathrm{LP} \_\mathrm{LZ}} \mathrm{LZ} \frac{\mathrm{LChl}}{\mathrm{LP}} \\
& -m_{\mathrm{PLChl}}-\tau(\mathrm{SD}+\mathrm{P}) \mathrm{LChl}
\end{aligned}
$$

3P1Z (includes SP, MP, LP, Z)

$$
\begin{aligned}
& \frac{\partial \mathrm{SP}}{\partial t}=\mu_{\mathrm{SP}} \mathrm{SP}-g_{\mathrm{SP} \_} \mathrm{Z}-m_{\mathrm{P}} \mathrm{SP}-\tau(\mathrm{SD}+\mathrm{P}) \mathrm{SP} \\
& -w_{\mathrm{SP}} \frac{\partial \mathrm{SP}}{\mathrm{d} z} \\
& \frac{\partial \mathrm{MP}}{\partial t}=\mu_{\mathrm{MP}} \mathrm{MP}-g_{\mathrm{MP} \_\mathrm{Z}}-m_{\mathrm{P}} \mathrm{MP}-\tau(\mathrm{SD}+\mathrm{P}) \mathrm{MP} \\
& -w_{\mathrm{MP}} \frac{\partial \mathrm{MP}}{\mathrm{d} z} \\
& \frac{\partial \mathrm{LP}}{\partial t}=\mu_{\mathrm{LP}} \mathrm{LP}-g_{\mathrm{LP}} \mathrm{Z} \mathrm{Z}-m_{\mathrm{P}} \mathrm{LP}-\tau(\mathrm{SD}+\mathrm{P}) \mathrm{LP} \\
& -w_{\mathrm{LP}} \frac{\partial \mathrm{LP}}{\mathrm{d} z} \\
& \frac{\partial \mathrm{Z}}{\partial t}=\left(g_{\mathrm{SP} \_\mathrm{Z}}+g_{\mathrm{MP} \_\mathrm{Z}}+g_{\mathrm{LP} \_\mathrm{Z}}\right) \beta \mathrm{Z}-l_{\mathrm{BM} \_\mathrm{Z}} \\
& -l_{\mathrm{E}} \frac{\mathrm{P}^{2}}{k_{\mathrm{P}}+\mathrm{P}^{2}} \beta \mathrm{Z}-m_{\mathrm{Z}} \mathrm{Z}^{2} \\
& \frac{\partial \mathrm{SD}}{\partial t}=\left(g_{\mathrm{SP} \_\mathrm{Z}}+g_{\mathrm{MP} \_\mathrm{Z}}+g_{\mathrm{LP}_{-} \mathrm{Z}}\right)(1-\beta) \mathrm{Z}+m_{\mathrm{Z}} \mathrm{Z}^{2}+m_{\mathrm{P}} \mathrm{P} \\
& -\tau(\mathrm{SD}+\mathrm{P}) \mathrm{SD}-r_{\mathrm{SD}} \mathrm{SD} \\
& -w_{\mathrm{SD}} \frac{\partial \mathrm{SD}}{\partial z} \\
& \frac{\partial \mathrm{LD}}{\partial t}=\tau(\mathrm{SD}+\mathrm{P})^{2}-r_{\mathrm{LD}} \mathrm{LD}-w_{\mathrm{LD}} \frac{\partial \mathrm{LD}}{\partial z} \\
& \frac{\partial \mathrm{NO} 3}{\partial t}=-\mu_{\mathrm{SP} \_\mathrm{NO} 3} \mathrm{P}-\mu_{\mathrm{MP} \_\mathrm{NO} 3} \mathrm{P}-\mu_{\mathrm{LP} \_\mathrm{NO}} \mathrm{P}+n \mathrm{NH} 4 \\
& \frac{\partial \mathrm{NH} 4}{\partial t}=-\mu_{\mathrm{SP} \_\mathrm{NH} 4} \mathrm{P}-\mu_{\mathrm{MP} \_\mathrm{NH} 4} \mathrm{P}-\mu_{\mathrm{LP} \_\mathrm{NH} 4} \mathrm{P}-n \mathrm{NH} 4 \\
& +l_{\mathrm{BM} \_\mathrm{ZZ}}+l_{\mathrm{E}} \frac{\mathrm{P}^{2}}{k_{\mathrm{P}}+\mathrm{P}^{2}} \beta \mathrm{Z}+r_{\mathrm{SD}} \mathrm{SD}+r_{\mathrm{LD}} \mathrm{LD} \\
& \frac{\partial \mathrm{SChl}}{\partial t}=\rho_{\mathrm{SChl}} \mu_{\mathrm{SP}} \mathrm{SChl}-g_{\mathrm{SP} \_\mathrm{ZZ}} \frac{\mathrm{SChl}}{\mathrm{SP}}-m_{\mathrm{P}} \mathrm{SChl} \\
& -\tau(\mathrm{SD}+\mathrm{P}) \mathrm{SChl}
\end{aligned}
$$

$$
\begin{aligned}
\frac{\partial \mathrm{MChl}}{\partial t} & =\rho_{\mathrm{MChl}} \mu_{\mathrm{MP}} \mathrm{MChl}-g_{\mathrm{MP}} \mathrm{ZZ} \frac{\mathrm{MChl}}{\mathrm{MP}}-m_{\mathrm{P}} \mathrm{MChl} \\
& -\tau(\mathrm{SD}+\mathrm{P}) \mathrm{MChl} \\
\frac{\partial \mathrm{LChl}}{\partial t} & =\rho_{\mathrm{LChl}} \mu_{\mathrm{LP}} \mathrm{LChl}-g_{\mathrm{LP}} \mathrm{Z} \mathrm{Z} \frac{\mathrm{LChl}}{\mathrm{LP}}-m_{\mathrm{P}} \mathrm{LChl} \\
& -\tau(\mathrm{SD}+\mathrm{P}) \mathrm{LChl}
\end{aligned}
$$

\section{P2Z (includes SP, MP, LP, SZ, LZ)}

$$
\begin{aligned}
& \frac{\partial \mathrm{SP}}{\partial t}=\mu_{\mathrm{SP}} \mathrm{SP}-g_{\mathrm{SP} \_\mathrm{SZ}} \mathrm{SZ}-g_{\mathrm{SP} \_\mathrm{LZ}} \mathrm{LZ}-m_{\mathrm{P}} \mathrm{SP} \\
& -\tau(\mathrm{SD}+\mathrm{P}) \mathrm{SP}-w_{\mathrm{SP}} \frac{\partial \mathrm{SP}}{\mathrm{d} z} \\
& \frac{\partial \mathrm{MP}}{\partial t}=\mu_{\mathrm{MP}} \mathrm{MP}-g_{\mathrm{MP} \_} \mathrm{SZ} \mathrm{SZ}-g_{\mathrm{MP} \_\mathrm{LZ} Z} \mathrm{LZ}-m_{\mathrm{P}} \mathrm{MP} \\
& -\tau(\mathrm{SD}+\mathrm{P}) \mathrm{MP}-w_{\mathrm{MP}} \frac{\partial \mathrm{MP}}{\mathrm{d} z} \\
& \frac{\partial \mathrm{LP}}{\partial t}=\mu_{\mathrm{LP}} \mathrm{LP}-g_{\mathrm{LP} \_\mathrm{SZ}} \mathrm{SZ}-g_{\mathrm{LP} \_\mathrm{LZ} Z} \mathrm{LZ}-m_{\mathrm{P}} \mathrm{LP} \\
& -\tau(\mathrm{SD}+\mathrm{P}) \mathrm{LP}-w_{\mathrm{LP}} \frac{\partial \mathrm{LP}}{\mathrm{d} z} \\
& \frac{\partial \mathrm{SZ}}{\partial t}=\left(g_{\mathrm{SP} \_} \mathrm{SZ}+g_{\mathrm{MP} \_\mathrm{SZ}}+g_{\mathrm{LP} \_} \mathrm{SZ}\right) \beta_{\mathrm{SZ}}-l_{\mathrm{BM} \_\mathrm{SZ}} \mathrm{SZ} \\
& -l_{\mathrm{E}} \frac{\mathrm{P}^{2}}{k_{\mathrm{P}}+\mathrm{P}^{2}} \beta_{\mathrm{SZ}}-m_{\mathrm{Z}} \mathrm{SZ}^{2} \\
& \frac{\partial \mathrm{LZ}}{\partial t}=\left(g_{\mathrm{SP} \_\mathrm{LZ}}+g_{\mathrm{MP} \_\mathrm{LZ}}+g_{\mathrm{LP} \_\mathrm{LZ}}\right) \beta_{\mathrm{LZ}}-l_{\mathrm{BM} \_\mathrm{LZ}} \mathrm{LZ} \\
& -l_{\mathrm{E}} \frac{\mathrm{P}^{2}}{k_{\mathrm{P}}+\mathrm{P}^{2}} \beta_{\mathrm{LZ}}-m_{\mathrm{Z}} \mathrm{LZ}{ }^{2} \\
& \frac{\partial \mathrm{SD}}{\partial t}=\left(g_{\mathrm{SP} \_\mathrm{SZ}}+g_{\mathrm{MP} \_\mathrm{SZ}}+g_{\mathrm{LP} \_\mathrm{SZ}}\right)(1-\beta) \mathrm{SZ} \\
& +\left(g_{\mathrm{SP} \_\mathrm{LZ}}+g_{\mathrm{MP} \_\mathrm{LZ}}+g_{\mathrm{LP} \_\mathrm{LZ}}\right)(1-\beta) \mathrm{LZ} \\
& +m_{\mathrm{Z}} \mathrm{SZ}^{2}+m_{\mathrm{Z}} \mathrm{LZ}^{2}+m_{\mathrm{P}} \mathrm{P}-\tau(\mathrm{SD}+\mathrm{P}) \mathrm{SD} \\
& -r_{\mathrm{SD}} \mathrm{SD}-w_{\mathrm{SD}} \frac{\partial \mathrm{SD}}{\partial z} \\
& \frac{\partial \mathrm{LD}}{\partial t}=\tau(\mathrm{SD}+\mathrm{P})^{2}-r_{\mathrm{LD}} \mathrm{LD}-w_{\mathrm{LD}} \frac{\partial \mathrm{LD}}{\partial z} \\
& \frac{\partial \mathrm{NO} 3}{\partial t}=-\mu_{\mathrm{SP} \_\mathrm{NO} 3} \mathrm{P}-\mu_{\mathrm{MP} \_\mathrm{NO} 3} \mathrm{P}-\mu_{\mathrm{LP} \_\mathrm{NO} 3} \mathrm{P}+n \mathrm{NH} 4 \\
& \frac{\partial \mathrm{NH} 4}{\partial t}=-\mu_{\mathrm{SP} \_\mathrm{NH} 4} \mathrm{P}-\mu_{\mathrm{MP}} \mathrm{NH}_{4} \mathrm{P}-\mu_{\mathrm{LP}} \mathrm{NH}_{4} \mathrm{P}-n \mathrm{NH} 4 \\
& +l_{\mathrm{BM} \_} \mathrm{SZ} \mathrm{SZ}+l_{\mathrm{BM} \_\mathrm{LZ}} \mathrm{LZ}+l_{\mathrm{E}} \frac{\mathrm{P}^{2}}{k_{\mathrm{P}}+\mathrm{P}^{2}} \beta_{\mathrm{Z}}+r_{\mathrm{SD}} \mathrm{SD} \\
& +r_{\mathrm{LD}} \mathrm{LD} \\
& \frac{\partial \mathrm{SChl}}{\partial t}=\rho_{\mathrm{SChl} l} \mu_{\mathrm{SP}} \mathrm{SChl}-g_{\mathrm{SP} \_\mathrm{SZ}} \mathrm{SZ} \frac{\mathrm{SChl}}{\mathrm{SP}}-g_{\mathrm{SP} \_\mathrm{LZ}} \mathrm{LZ} \frac{\mathrm{SChl}}{\mathrm{SP}} \\
& -m_{\mathrm{P}} \mathrm{SChl}-\tau(\mathrm{SD}+\mathrm{P}) \mathrm{SChl}
\end{aligned}
$$




$$
\begin{aligned}
\frac{\partial \mathrm{MChl}}{\partial t} & =\rho_{\mathrm{MChl}} \mu_{\mathrm{MP}} \mathrm{MChl}-g_{\mathrm{MP} \_\mathrm{SZ}} \mathrm{SZ} \frac{\mathrm{SChl}}{\mathrm{MP}} \\
& -g_{\mathrm{MP} \_\mathrm{LZ}} \mathrm{LZ} \frac{\mathrm{MChl}}{\mathrm{MP}}-m_{\mathrm{P} M C h l}-\tau(\mathrm{SD}+\mathrm{P}) \mathrm{MChl} \\
\frac{\partial \mathrm{LChl}}{\partial t} & =\rho_{\mathrm{LChl}} \mu_{\mathrm{LP}} \mathrm{LChl}-g_{\mathrm{LP} \_\mathrm{SZ}} \mathrm{SZ} \frac{\mathrm{LChl}}{\mathrm{LP}}-g_{\mathrm{LP} \_\mathrm{LZ} L Z} \frac{\mathrm{LChl}}{\mathrm{LP}} \\
& -m_{\mathrm{PLChl}}-\tau(\mathrm{SD}+\mathrm{P}) \mathrm{LChl}
\end{aligned}
$$


Acknowledgements. This work was supported by the NASA Earth and Space Science Fellowship Program (grant NNX10AN50H) and the NASA Interdisciplinary Science Program (NNX11AD47G). The authors are grateful to the NASA US Eastern Continental Shelf Carbon Cycling (USECoS) team for their useful comments, especially Kimberly Hyde, Antonio Mannino and Xiaoju Pan for providing the satellite-derived size-fractionated chlorophyll data. The authors would like to thank the SeaWiFS Project (code 970.2) and the Distributed Active Archive Center (code 610.2) at the Goddard Space Flight Center, Greenbelt, MD 20771, for the production and distribution of these data, respectively. This is VIMS contribution no. 3342.

Edited by: K. Fennel

\section{References}

Anderson, T. R.: Plankton functional type modelling: running before we can walk?, J. Plankton Res., 27, 1073-1081, doi:10.1093/plankt/fbi076, 2005.

Baird, M. E. and Suthers, I. M.: Increasing model structural complexity inhibits the growth of initial condition errors, Ecol. Complex., 7, 478-486, doi:10.1016/j.ecocom.2009.12.001, 2010.

Bagniewski, W., Fennel, K., Perry, M. J., and D’Asaro, E. A.: Optimizing models of the North Atlantic spring bloom using physical, chemical and bio-optical observations from a Lagrangian float, Biogeosciences, 8, 1291-1307, doi:10.5194/bg-8-12912011, 2011.

Costanza, R. and Sklar, F. H.: Articulation, accuracy and effectiveness of mathematical models: A review of freshwater wetland applications, Ecol. Model., 27, 45-68, doi:10.1016/03043800(85)90024-9, 1985.

Denman, K. L.: Modelling planktonic ecosystems: Parameterizing complexity, Progr. Oceanogr., 57, 429-452, 2003.

Fan, W. and Lv, X.: Data assimilation in a simple marine ecosystem model based on spatial biological parameterizations, Ecol. Model., 220, 1997-2008, doi:10.1016/j.ecolmodel.2009.04.050, 2009.

Fennel, K., Wilkin, J., Levin, J., Moisan, J., O’Reilly, J., and Haidvogel, D.: Nitrogen cycling in the Middle Atlantic Bight: Results from a three-dimensional model and implications for the North Atlantic nitrogen budget, Global Biogeochem. Cy., 20, GB3007, doi:10.1029/2005gb002456, 2006.

Flynn, K. J.: Castles built on sand: dysfunctionality in plankton models and the inadequacy of dialogue between biologists and modellers, J. Plankton Res., 27, 1205-1210, 2005.

Follows, M. J., Dutkiewicz, S., Grant, S., and Chisholm, S. W.: Emergent biogeography of microbial communities in a model ocean, Science, 315, 1843-1846, doi:10.1126/science.1138544, 2007.

Friedrichs, M. A. M.: The assimilation of SeaWiFS and JGOFS EqPac data into a marine ecosystem model of the central equatorial Pacific, Deep-Sea Res. Pt. II, 49, 289-319, 2002.

Friedrichs, M. A. M., Hood, R. R., and Wiggert, J. D.: Ecosystem model complexity versus physical forcing: Quantification of their relative impact with assimilated Arabian Sea data, Deep-Sea Res. Pt. II, 53, 576-600, doi:10.1016/j.dsr2.2006.01.026, 2006.

Friedrichs, M. A. M., Dusenberry, J., Anderson, L., Armstrong, R., Chai, F., Christian, J.,Doney, S. C., Dunne, J., Fujii, M.,
Hood, R., McGillicuddy, D., Moore, K., Schartau, M., Sptiz, Y. H., and Wiggert, J.: Assessment of skill and portability in regional marine biogeochemical models: role of multiple phytoplankton groups, J. Geophys. Res., 112, C08001, doi:10.1029/2006JC003852, 2007.

Friedrichs, M. A. M., Carr, M.-E., Barber, R., Scardi, M., Antoine, D., Armstrong, R. A., Asanuma, I., Behrenfeld, M. J., Buitenhuis, E. T., Chai, F., Christian, J. R., Ciotti, A. M., Doney, S., C., Dowell, M., Dunne, J., Gentili, B., Gregg, W., Hoepffner, N., Ishizaka, J., Kameda, T., Lima, I., Marra, J., Mélin, F., Moore, J. K., Morel, A., O'Malley, R. T., O'Reilly, J., Saba, V. S., Schmeltz, M., Smyth, T. J., Tjiputra, J., Waters, K., Westberry, T. K., and Winguth, A.: Assessing the uncertainties of model estimates of primary productivity in the tropical Pacific Ocean, J. Mar. Syst., 76, 113-133, doi:10.1016/j.jmarsys.2008.05.010, 2009.

Fulton, E. A., Smith, A. D. M., and Johnson, C. R.: Effect of complexity on marine ecosystem models, Mar. Ecol.-Prog. Ser., 253, 1-16, doi:10.3354/meps253001, 2003.

Garcia-Gorriz, E., Hoepffner, N., and Ouberdous, M.: Assimilation of SeaWiFS data in a coupled physical-biological model of the Adriatic Sea, J. Mar. Syst., 40, 233-252, doi:10.1016/s09247963(03)00020-4, 2003.

Giering, R. and Kaminski, T.: Recipes for adjoint code construction, ACM T. Math. Software, 24, 437-474, doi:10.1145/293686.293695, 1998.

Gilbert, J. C. and Lemarechal, C.: Some numerical experiments with variable-storage quasi-newton algorithms, Math. Program., 45, 405-435, 1989.

Gregg, W., Friedrichs, M. A. M., Robinson, A. R., Rose, K., Schlitzer, R., and Thompson, K. R.: Skill assessment in ocean biological data assimilation, J. Mar. Syst., 76, 16-33, doi:10.1016/j.jmarsys.2008.05.006, 2009.

Hannah, C., Vezina, A., and St John, M.: The case for marine ecosystem models of intermediate complexity, Progr. Oceanogr., 84, 121-128, doi:10.1016/j.pocean.2009.09.015, 2010.

Hashioka, T., Vogt, M., Yamanaka, Y., Le Quéré, C., Buitenhuis, E. T., Aita, M. N., Alvain, S., Bopp, L., Hirata, T., Lima, I., Sailley, S., and Doney, S. C.: Phytoplankton competition during the spring bloom in four plankton functional type models, Biogeosciences, 10, 6833-6850, doi:10.5194/bg-10-6833-2013, 2013.

Hemmings, J. C. P., Srokosz, M. A., Challenor, P., and Fasham, M. J. R.: Split-domain calibration of an ecosystem model using satellite ocean colour data, J. Mar. Syst., 50, 141-179, doi:10.1016/j.jmarsys.2004.02.003, 2004.

Hofmann, E., Druon, J.-N. Fennel, K., Friedrichs, M., Haidvogel, D., Lee, C., Mannino, A., McClain, C., Najjar, R., O'Reilly, J., Pollard, D., Previdi, M., Seitzinger, S., Siewert, J., Signorini, S., and Wilkin, J.: Eastern U.S. continental shelf carbon budget: integrating models, data assimilation, and analysis, Oceanography, 21, 86-104, 2008.

Hofmann, E. E., Cahill, B., Fennel, K., Friedrichs, M. A. M., Hyde, K., Lee, C., Mannino, A., Najjar, R. G., O'Reilly, J. E., Wilkin, J., and Xue, J.: Modeling the dynamics of continental shelf carbon, Annu. Rev. Mar. Sci., 3, 93-122, doi:10.1146/annurev-marine120709-142740, 2011.

Jolliff, J. K., Kindle, J. C., Shulman, I., Penta, B., Friedrichs, M. A. M., Helber, R., and Arnone, R. A: Summary diagrams for cou- 
pled hydrodynamic-ecosystem model skill assessment, J. Mar. Syst., 76, 64-82, doi:10.1016/j.jmarsys.2008.05.014, 2009.

Kishi, M. J., Kashiwai, M., Ware, D. M., Megrey, B. A., Eslinger, D. L., Werner, F. E., Noguchi-Aita, F. M., Azumaya, T., Fujii, M., Hashimoto, S., Huang, D. J., Iizumi, H., Ishida, Y., Kang, S., Kantakov, G. A., Kim, H. C., Komatsu, K., Navrotsky, V. V., Smith, S. L., Tadokoro, K., Tsuda, A., Yamamura, O., Yamanaka, Y., Yokouchi, K., Yoshie, N., Zhang, J., Zuenko, Y. I., and Zvalinsky, V. I.: NEMURO - a lower trophic level model for the North Pacific marine ecosystem, Ecol. Model., 202, 12-25, doi:10.1016/j.ecolmodel.2006.08.021, 2007.

Lawson, L. M., Spitz, Y. H., Hofmann, E. E., and Long, R. B.: A data assimilation technique applied to a predator-prey model, B. Math. Biol., 57, 593-617, doi:10.1016/S0092-8240(05)80759-1, 1995.

Lawson, L. M., Hofmann, E. E., and Spitz, Y. H.: Time series sampling and data assimilation in a simple marine ecosystem model, Deep-Sea Res. Pt. II, 43, 625-651, doi:10.1016/09670645(95)00096-8, 1996.

Lehmann, M. K., Fennel, K., and He, R.: Statistical validation of a 3-D bio-physical model of the western North Atlantic, Biogeosciences, 6, 1961-1974, doi:10.5194/bg-6-1961-2009, 2009.

McDonald, C. P., Bennington, V., Urban, N. R., and McKinley, G. A.: 1-D test-bed calibration of a 3-D Lake Superior biogeochemical model, Ecol. Model., 225, 115-126, doi:10.1016/j.ecolmodel.2011.11.021, 2012.

Pan, X., Mannino, A., Russ, M. E., Hooker, S. B., and Harding Jr., L. W.: Remote sensing of phytoplankton pigment distribution in the United States northeast coast, Remote Sens. Environ., 114, 2403-2416, doi:10.1016/j.rse.2010.05.015, 2010.

Paudel, R. and Jawitz, J. W.: Does increased model complexity improve description of phosphorus dynamics in a large treatment wetland?, Ecol. Eng., 42, 283-294, doi:10.1016/j.ecoleng.2012.02.014, 2012.

Raick, C., Soetaert, K., and Gregoire, M.: Model complexity and performance: How far can we simplify?, Progr. Oceanogr., 70, 27-57, doi:10.1016/j.pocean.2006.03.001, 2006.
Rykiel, E. J.: Testing ecological models: The meaning of validation, Ecol. Model., 90, 229-244, doi:10.1016/0304-3800(95)00152-2, 1996.

Salihoglu, B. and Hofmann, E. E.: Simulations of phytoplankton species and carbon production in the equatorial Pacific Ocean 1. Model configuration and ecosystem dynamics, J. Mar. Res., 65, 219-273, 2007.

Stow, C. A., Jolliff, J., McGillicuddy Jr., D. J., Doney, S. C., Allen, J. I., Friedrichs, M. A. M., Rose, K. A., and Wallhead, P.: Skill assessment for coupled biological/physical models of marine systems, J. Mar. Syst., 76, 4-15, doi:10.1016/j.jmarsys.2008.03.011, 2009.

Stramska, M. and Stramski, D.: Variability of particulate organic carbon concentration in the north polar Atlantic based on ocean color observations with Sea-viewing Wide Field-ofview Sensor (SeaWiFS), J. Geophys. Res.-Oceans, 110, C10018, doi:10.1029/2004jc002762, 2005.

Tjiputra, J. F., Polzin, D., and Winguth, A. M. E.: Assimilation of seasonal chlorophyll and nutrient data into an adjoint threedimensional ocean carbon cycle model: Sensitivity analysis and ecosystem parameter optimization, Global Biogeochem. Cy., 21, GB1001, doi:10.1029/2006gb002745, 2007.

Ward, B. A., Friedrichs, M. A. M., Anderson, T. R., and Oschlies, A.: Parameter optimisation techniques and the problem of underdetermination in marine biogeochemical models, J. Mar. Syst., 81, 34-43, doi:10.1016/j.jmarsys.2009.12.005, 2010.

Ward, B. A., Schartau, M., Oschlies, A., Martin, A. P., Follows, M. J., and Anderson, T. R.: When is a biogeochemical model too complex? Objective model reduction and selection for North Atlantic time-series sites, Progr. Oceanogr., 116, 49-65, doi:10.1016/j.pocean.2013.06.002, 2013.

Xiao, Y. and Friedrichs, M. A. M.: The assimilation of satellitederived data into a one-dimensional lower trophic level marine ecosystem model, J. Geophys. Res.-Oceans, 119, 2691-2712, doi:10.1002/2013JC009433, 2014. 